\title{
Exophilin-5 regulates allergic airway inflammation by controlling IL-33-mediated Th2 responses
}

\author{
Katsuhide Okunishi, ${ }^{1}$ Hao Wang, ${ }^{1}$ Maho Suzukawa, ${ }^{2,3}$ Ray Ishizaki, ${ }^{1}$ Eri Kobayashi, ${ }^{1}$ Miho Kihara, ${ }^{4}$ Takaya Abe, ${ }^{4,5}$ \\ Jun-ichi Miyazaki, ${ }^{6}$ Masafumi Horie, ${ }^{7}$ Akira Saito, ${ }^{7}$ Hirohisa Saito, ${ }^{8}$ Susumu Nakae, ${ }^{9}$ and Tetsuro Izumi'
}

\begin{abstract}
'Laboratory of Molecular Endocrinology and Metabolism, Department of Molecular Medicine, Institute for Molecular and Cellular Regulation, Gunma University, Maebashi, Japan. ²National Hospital Organization Tokyo National Hospital, Tokyo, Japan. ${ }^{3}$ Division of Respiratory Medicine and Allergology, Department of Medicine, Teikyo University School of Medicine, Tokyo, Japan. ${ }^{4}$ Laboratory for Animal Resource Development and ${ }^{5}$ Genetic Engineering, RIKEN Center for Biosystems Dynamics Research, Kobe, Japan. ${ }^{6}$ Institute of Scientific and Industrial Research, Osaka University, Osaka, Japan. ${ }^{7}$ Department of Respiratory Medicine, Graduate School of Medicine, The University of Tokyo, Tokyo, Japan. ${ }^{8}$ Department of Allergy and Clinical Immunology, National Research Institute for Child Health and Development, Tokyo, Japan. ' Laboratory of Systems Biology, Center for Experimental Medicine and Systems Biology, Institute of Medical Science, The University of Tokyo, Tokyo, Japan.
\end{abstract}

\begin{abstract}
A common variant in the RAB27A gene in adults was recently found to be associated with the fractional exhaled nitric oxide level, a marker of eosinophilic airway inflammation. The small GTPase Rab27 is known to regulate intracellular vesicle traffic, although its role in allergic responses is unclear. We demonstrated that exophilin-5, a Rab27-binding protein, was predominantly expressed in both of the major IL-33 producers, lung epithelial cells, and the specialized IL-5 and IL-13 producers in the $\mathrm{CD} 44^{\text {hi }} \mathrm{CD} 62 \mathrm{~L}^{10} \mathrm{CXCR} 3^{10}$ pathogenic Th2 cell population in mice. Exophilin-5 deficiency increased stimulant-dependent damage and IL-33 secretion by lung epithelial cells. Moreover, it enhanced IL-5 and IL-13 production in response to TCR and IL-33 stimulation from a specific subset of pathogenic Th2 cells that expresses a high level of IL-33 receptor, which exacerbated allergic airway inflammation in a mouse model of asthma. Mechanistically, exophilin-5 regulates extracellular superoxide release, intracellular ROS production, and phosphoinositide 3-kinase activity by controlling intracellular trafficking of Nox2containing vesicles, which seems to prevent the overactivation of pathogenic Th2 cells mediated by IL-33. This is the first report to our knowledge to establish the significance of the Rab27-related protein exophilin-5 in the development of allergic airway inflammation, and provides insights into the pathophysiology of asthma.
\end{abstract}

\section{Introduction}

Allergic asthma is a chronic inflammatory airway disease characterized by airway eosinophilia, mucus hypersecretion, and airway hyperresponsiveness (1). Its incidence is steadily increasing, with an estimated 339 million people affected worldwide (2). The most common treatment is topical inhaled corticosteroid application, which, while effective, is associated with corticosteroid nonspecific suppression of immune responses, resulting in increased incidence of infections such as esophageal candidiasis. Further, steroids are only temporarily effective and do not provide a cure for asthma. To move toward a cure, we must better understand the underlying pathophysiology of this process.

A defining feature of allergic responses, such as in asthma, is an antigen-specific Th2-dominant immune response, which can develop through interactions among many different types of cells. It is well established that biologically active substances, such as cytokines, play crucial roles, and allergy research has strongly

\section{Related Commentary: p. 3419}

Authorship note: $\mathrm{KO}$ and $\mathrm{HW}$ contributed equally to this work. Conflict of interest: The authors have declared that no conflict of interest exists. Copyright: () 2020, American Society for Clinical Investigation. Submitted: January 30, 2019; Accepted: March 31, 2020; Published: June 8, 2020. Reference information: / Clin Invest. 2020;130(7):3919-3935. https://doi.org/10.1172/JCl127839. focused on the functions of such biologically active substances and their producers in the etiology of allergy. On the other hand, although biological substances including cytokines are released from their producer cells extracellularly to engage their target cells, our understanding of the role of secretory machinery in allergy has advanced little.

Secretory machinery is classified into 2 pathways: constitutive and regulated. In the former, biologically active molecules are released consecutively upon their synthesis. In the latter, molecules are preformed, stored in vesicles, and rapidly released upon stimulation, and comprises a highly developed regulatory pathway in higher organisms that is essential for rapid intercellular communication. Rab27 is a small GTPase belonging to the Rab superfamily, and is well recognized as an essential player in regulated secretion (3). The role of Rab27a in insulin secretion by pancreatic $\beta$ cells (4), as well as in melanosome transfer from melanocytes to keratinocytes (5), is well established. It is also known to positively regulate secretion of cytotoxic granules by cytotoxic cells such as NK cells and cytotoxic T cells $(6,7)$, and mutation of Rab27a in humans results in type II Griscelli syndrome, one of the familial hemophagocytic syndromes accompanied by immune dysfunction and albinism (8). In addition, Rab27a regulates cell functions by controlling intracellular trafficking of NADPH oxidase-containing vesicles and subsequent intracellular ROS production in macrophages and neutrophils $(9,10)$. On the other hand, although it is reported that Rab27 is expressed in other immune cells such 
as mast cells where it regulates exocytosis $(11,12)$, dendritic cells (DCs) (13), Th cells (7), and lung epithelial cells (14), all of which play essential roles in antigen-specific allergic immune responses in the lung, the roles of Rab27 in the net allergic immune responses in vivo are unknown. A recent study showed a link between RAB27A single nucleotide polymorphism (SNP) and fractional exhaled nitric oxide (FeNO) levels (15), an indicator of allergic airway inflammation (16), suggesting the involvement of Rab27 in the regulation of allergic immune responses.

Eleven effectors have been shown to bind the active, GTPbound form of Rab27 and regulate intracellular vesicle traffic at specific steps (3). Among these 11 Rab27 effectors, the present study focused on exophilin-5, a large protein composed of 1960 and 1989 amino acids in mice and humans, respectively. Compared with the other Rab27 effectors, its biological function remains obscure, and few studies have investigated its biological functions. For example, in humans, mutation of the exophilin-5encoding EXPH5 gene causes skin fragility, which results in the development of epidermolysis bullosa (17). In cells, exophilin-5 is reported to positively regulate exosome secretion in HeLa cells (18) and intracellular trafficking of PI4-kinase type 2a-containing late endosomes in the human T cell leukemia cell line Jurkat (19). Moreover, a recent study revealed that exophilin-5 is present in a human Th2-enriched memory-type $\mathrm{CD} 4^{+}$cell fraction (20). However, the role of exophilin-5 in asthmatic responses is unclear. In the present study, using newly developed exophilin-5-knockout (Exph5-KO) mice and mouse models of asthma, we uncovered a role of exophilin- 5 as a regulator of IL-33 signaling in antigeninduced allergic immune responses.

\section{Results}

Lack of exophilin-5 exacerbates OVA-induced allergic inflammation. Because asthma is a well-established allergic disease in which CD $4^{+}$Th cells play essential roles, and exophilin- 5 is reported to be expressed in the human $\mathrm{CD} 4^{+} \mathrm{T}$ cell line Jurkat (19) and human Th2-enriched $\mathrm{CD}^{+}$cell fraction (20), we investigated the roles of exophilin-5 in antigen-induced allergic airway inflammation using newly generated Exph5-KO mice (Figure 1A). Exph5-KO mice were viable and fertile, showed no gross phenotypic abnormalities in body weight, growth, organ structures, or immune cell composition in peripheral lymph tissues, and did not spontaneously develop eosinophilic lung inflammation (Supplemental Figure 1A; supplemental material available online with this article; https:/doi. org/10.1172/JCI127839DS1). We first confirmed that OVA/alum sensitization alone in the absence of airway OVA challenges did not cause eosinophilic lung inflammation either in WT mice or in Exph5-KO mice (Supplemental Figure 1B). Next, spleen cells and thoracic lymph nodes were isolated from mice 7 days after the second OVA/alum injection and 24 hours after the final airway challenge, respectively, and were cultured in the presence and absence of OVA. In response to this ex vivo OVA restimulation, cells of both types from Exph5-KO mice produced higher amounts of typical Th2 cytokines IL-4, IL-5, and IL-13, especially IL-5 with a statistical significance, but not the typical Th1 cytokine IFN- $\gamma$, compared with those from WT mice (Figure 1, B and C, and Supplemental Figure 1, C and D). Consistently, levels of IL-5 and IL-13 (Figure 1D and Supplemental Figure 1E), eosinophil infiltration (Figure 1E), and mucus production (Figure $1 \mathrm{~F}$ ) were markedly enhanced in the lungs of Exph5-KO mice after 3 days of OVA challenges. Airway hyperresponsiveness also tended to be enhanced in Exph5-KO mice (Figure 1G). These results suggest that systemic exophilin-5 deficiency enhances OVA-induced allergic airway inflammation, possibly through upregulation of OVA-specific Th2 cytokine production by immune cells.

Exophilin-5 deficiency in hematopoietic cells is essential for induction of enhanced allergic airway inflammation. Because exophilin-5 is reported to be expressed in both hematopoietic and nonhematopoietic cells $(17,19,20)$, we next used bone marrowchimeric (BM-chimeric) mice to identify the cells responsible for the phenotypes observed in Exph5-KO mice in an OVA-induced mouse model of asthma. Although exophilin-5 deficiency in BM cells did not affect the induction of OVA-specific Th2-type immune responses, exophilin-5 deficiency in recipient mice tended to enhance it (Figure 2A). However, exophilin-5 deficiency in BM cells significantly exacerbated eosinophilic lung inflammation to a level similar to that induced by exophilin- 5 deficiency in the whole body (Figure 2B), along with enhanced mucus production (Figure 2C). We confirmed that BM cell transplantation itself did not cause spontaneous development of eosinophilic lung inflammation in the absence of OVA antigen inhalation (Supplemental Figure 1F). These results indicate that lack of exophilin-5 in hematopoietic cells is sufficient to phenocopy the exacerbated allergic airway inflammation in Exph5-KO mice without affecting antigen sensitization induced by intraperitoneal antigen injections. The finding that exophilin-5 deficiency in recipient mice, regardless of types of transplanted $\mathrm{BM}$, tended to affect both the antigen sensitization phase and subsequent eosinophilic airway inflammation (Figure 2, A and B) suggests that exophilin-5 deficiency in structural cells also plays some roles in exacerbation of asthmatic phenotypes in Exph5-KO mice.

Exophilin-5 deficiency enhances IL-33 secretion by epithelial cells. Because exophilin-5 deficiency has genetically been demonstrated to induce fragility of keratinocytes in humans (17), we first examined the roles of exophilin-5 in nonhematopoietic immune cells. Epithelial cells of organs exposed to the external environment (e.g., lungs) are well recognized to modulate allergic immune responses by secreting cytokines such as IL-33 that can be released upon epithelial cell damage (21). Thus, we investigated whether exophilin-5 deficiency enhances IL-33 secretion upon exposure to external stimuli. Although IL-33 levels in bronchoalveolar lavage fluid (BALF) after harmless saline injection did not differ between WT and Exph5-KO mice, IL-33 secretion as well as lactate dehydrogenase (LDH) secretion into alveolar spaces after a single intratracheal injection of external, harmful Alternaria extract was significantly enhanced in Exph5-KO mice (Figure 3A). Among epithelial cell-derived cytokines, IL-33 was the major cytokine rapidly released after Alternaria stimulation. Antigen inhalation also induces rapid release of endogenous chemical mediators such as proteases and tumor necrosis factor $\alpha$ by mast cells, which potentially damages epithelial cells in the lungs of antigen-sensitized individuals $(22,23)$. In fact, after the third OVA aerosol challenge, although IL-33 levels in the lung homogenates were comparable between WT and Exph5-KO mice, rapid IL-33 release into the alveolar space was observed in Exph5-KO mice, but not in WT mice 
A Generation onf exophilin-5 knockout (Exph5 KO) mice

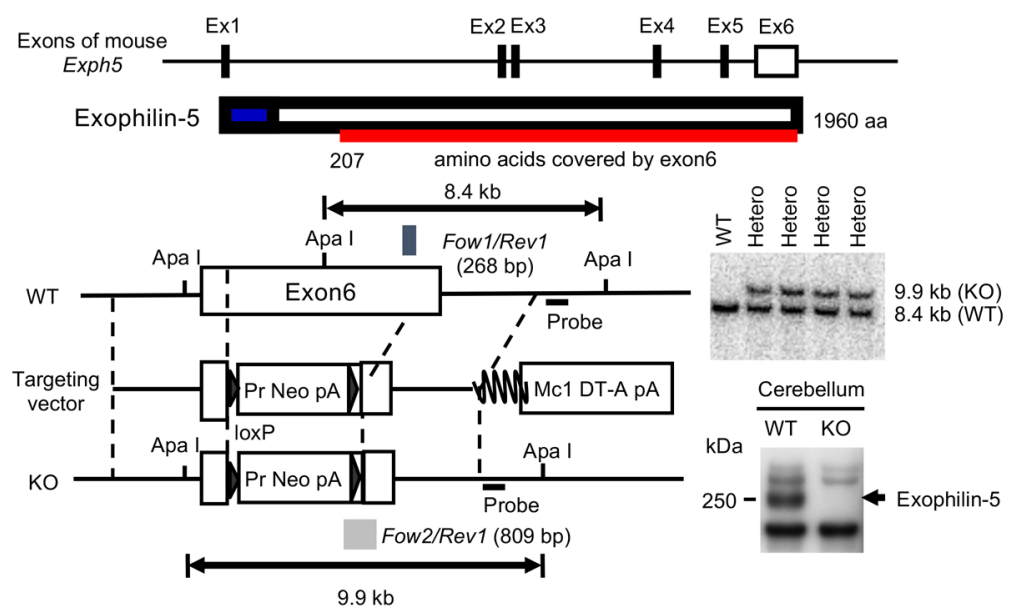

B

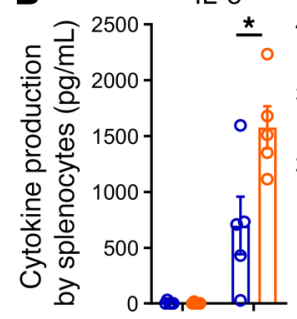

(-) OVA
IL-13

OWT

O Exph5 KO

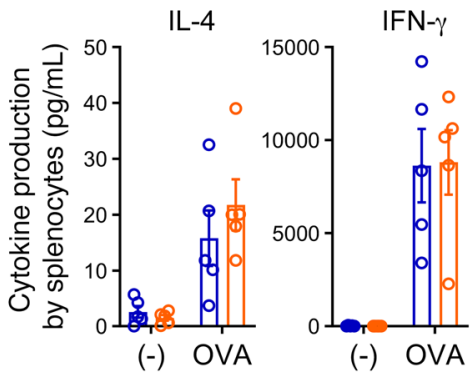

D

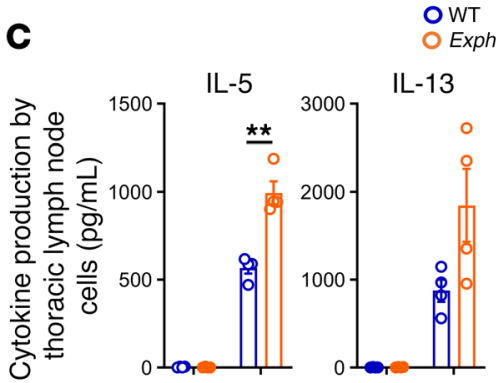

(-) OVA

(-) OVA

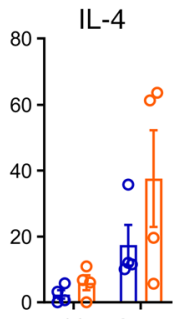

(-) OVA

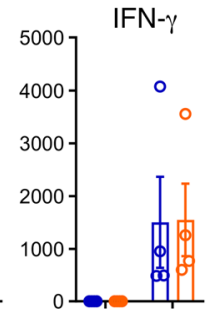

(-) OVA

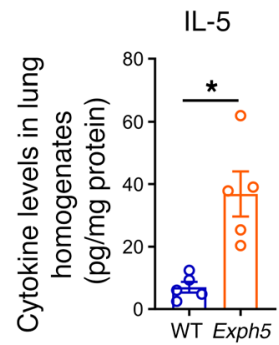

WT Exph

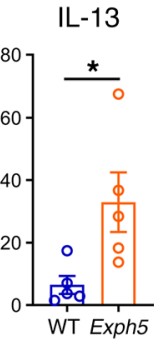

WT Exph5

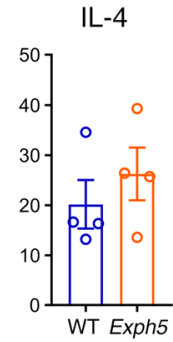

WT Exph5
IFN- $\gamma$

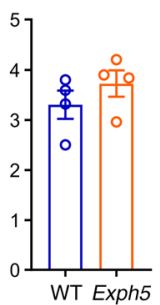

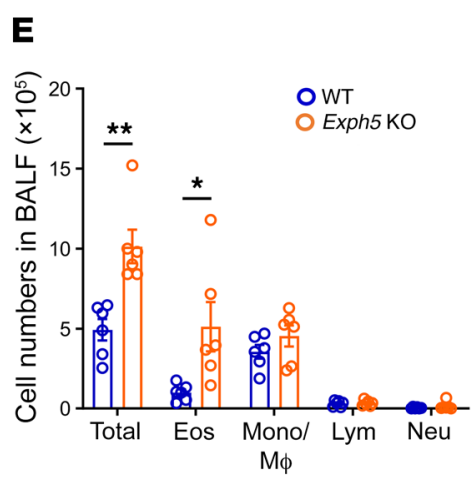
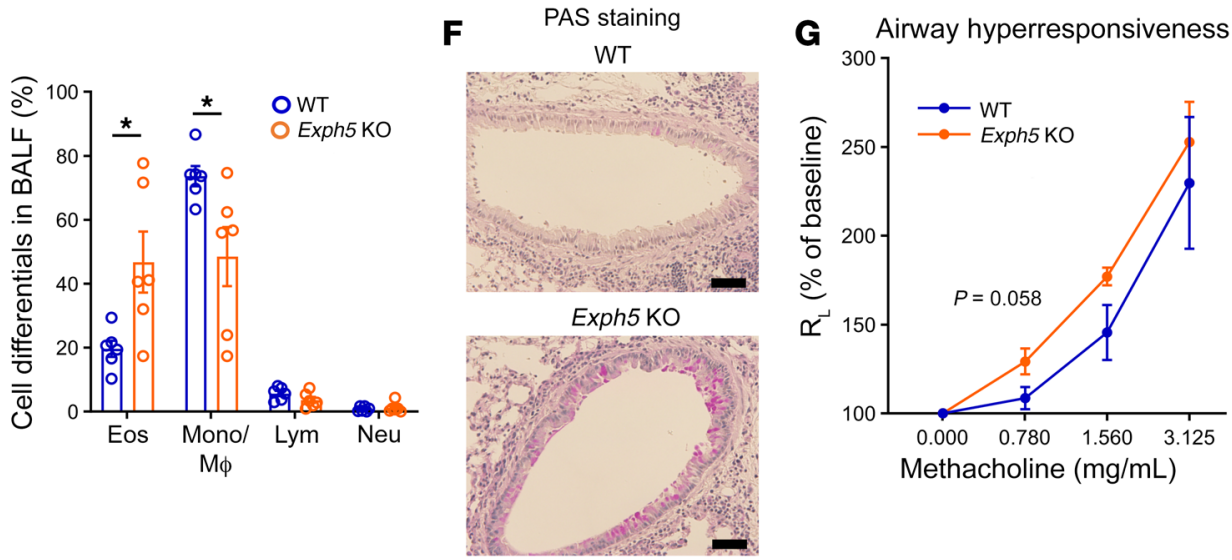

Figure 1. Exophilin-5 deficiency exacerbates lung inflammation in an OVA-induced mouse model of asthma. (A) Targeted disruption of the exophilin-5 gene on mouse chromosome 9 . The blue region in the amino acid sequence of exophilin-5 indicates the Rab-binding domain. Dark and light gray boxes indicate regions used for PCR genotyping of WT and KO alleles, respectively. Middle insert: Genomic Southern hybridization analysis of the backcrossed progenies from a cross of chimeric mice with C57BL/6 mice. The location of the external probe is shown with horizontal closed boxes in the upper panel. The probe hybridizes to Apal fragments of $9.9 \mathrm{~kb}$ and $8.4 \mathrm{~kb}$ from WT and KO alleles, respectively. Bottom insert: Expression of exophilin-5 protein in cerebellum from WT and exophilin-5-knockout (Exph5-KO) mice. A blot representative of 3 independent experiments with similar results is shown. (B-D) Phenotypes of Exph5-KO mice in an OVA-induced mouse model of asthma. (B and C) Cytokine production in response to ex vivo OVA restimulation (1 mg/ $\mathrm{mL}$ ) by splenocytes $(\mathbf{B})$ and by lung lymph node cells $(\mathbf{C})$. For dose- and time-dependent cytokine production by these cells, refer to Supplemental Figure 1, C and D. (D) Cytokine levels in the lungs of mice after 3-day OVA aerosol challenges. (E) Cell numbers and differentials in BALF after 3-day 3\% OVA inhalation. Cell differentials were determined by counting of cytospin samples stained with Diff-Quik. $n=4-6$ mice combined from at least 2 independent experiments in B-E. (F) Mucus production by the epithelia was assessed by PAS staining of sliced paraffin-fixed lung sections. An image representative of 6 samples from 2 independent experiments is shown. Scale bars: $50 \mu \mathrm{m}$. (G) Airway hyperresponsiveness assessed as change in lung resistance $\left(R_{L}\right)$ in response to methacholine. $n=6$ mice combined from 2 independent experiments. ${ }^{*} P<0.05 ;{ }^{* *} P<0.01$ by unpaired $t$ test. 

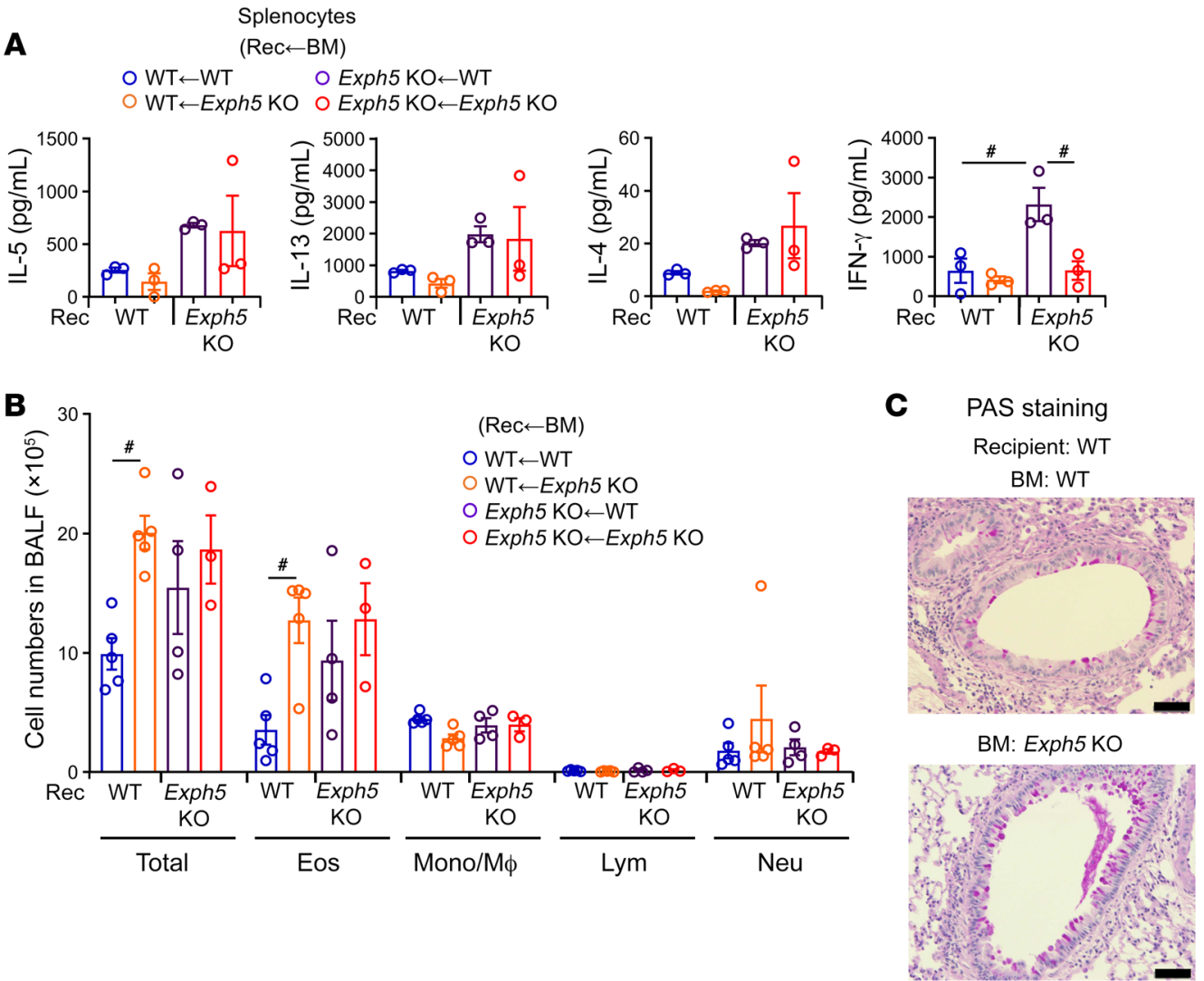

Figure 2. Phenotypes of BMchimeric mice in an OVAinduced mouse model of asthma. BM-chimeric mice generated as described in Methods were used in an OVA-induced mouse model of asthma. (A) Production of typical Th2 and Th1 cytokines by splenocytes obtained from OVA-sensitized mice in response to ex vivo OVA restimulation. Splenocytes were isolated from mice and were restimulated with ex vivo OVA as in Figure 1B ( $n=3$ mice in 1 cohort representative of 2 independent cohorts with similar results). (B) Cell numbers and differentials in BALF after 3-day 3\% OVA inhalation ( $n=3-5$ mice combined from 2 independent experiments). (C) Mucus production assessed by PAS staining. An image representative of 3 mice per group in 1 experiment is shown. Scale bars: $50 \mu \mathrm{m}$. ${ }^{\# P}<$ 0.05 by 1-way ANOVA with Tukey's post hoc test. Rec, recipient.
(Figure 3B). We confirmed that exophilin-5 deficiency in BM cells did not enhance IL-33 secretion (Figure 3C), suggesting that the enhanced IL-33 secretion in Exph5-KO mice was not due to alteration in secretion from local hematopoietic cells, such as mast cells. These results suggest that lung epithelial cells in Exph5-KO mice are more vulnerable to external stimuli and augment allergic lung inflammation through their enhanced IL-33 release. CD 45 CD326 ${ }^{+}$alveolar epithelial cells expressed exophilin-5 at a level equivalent to that in epidermal cells (Figure $4 \mathrm{~A}$ ), which are reported to express exophilin-5 in humans (17).

Exophilin-5 regulates responsiveness to IL-33 in pathogenic Th2 cells. We next investigated the mechanism by which exophilin- 5 deficiency in hematopoietic cells exacerbates OVA-induced allergic lung inflammation. Endo et al. recently reported that, among 4 fractions divided by CXCR3 and CD62L expression levels of effector cytokine-producing splenic CD $44^{\text {hi }}$ memory $\mathrm{T}$ cells, the $\mathrm{CD} 2 \mathrm{~L}^{\text {lo }} \mathrm{CXCR} 3^{\text {lo }}$ population selectively produces IL-5 and IL-13 proteins upon T cell receptor (TCR) stimulation (24). On the other hand, IL- 4 and IFN- $\gamma$ are produced by multiple populations. They also showed that the $\mathrm{CD} 62 \mathrm{~L}^{\text {lo }} \mathrm{CXCR} 3^{\text {lo }}$ population is essential for the development of antigen-dependent allergic lung inflammation, and named those cells pathogenic Th2 cells (25). Based on these precedents, we sorted these 4 fractions of CD $44^{\text {hi }}$ splenic memory-type $\mathrm{T}$ cells to examine the expression level of exophilin-5 in each fraction (Figure $4 \mathrm{~A}$ ). Splenic CD $4^{+}$Th cells were first enriched using microbead-conjugated anti-CD4. Then, 4 populations in $\mathrm{CD}^{+} \mathrm{CD}^{+} \mathrm{CD} 44^{\mathrm{hi}}$ memory $\mathrm{T}$ cells were sorted based on levels of cell surface CD62L and CXCR3. Among hematopoietic cells, Exph5 mRNA was highly expressed in 2 fractions of CD $44^{\text {hi }}$ memory-type CD4 ${ }^{+}$T cells: CD $62 \mathrm{~L}^{\text {lo }} \mathrm{CXCR} 3^{\text {lo }}$ and CD $62 \mathrm{~L}^{\text {hi }} \mathrm{CXCR} 3^{\text {lo }}$. Exophilin-5 deficiency did not alter expression levels of other 10 Rab27 effectors in either $\mathrm{CD}^{+} \mathrm{T}$ cells or pathogenic Th2 cells (Supplemental Figure 2A). We next tested whether the secretion of IL-5 and IL-13 was affected by exophilin-5 deficiency. In subsequent experiments, to avoid unnecessary stimulation of $\mathrm{T}$ cells and interference with plate-coated anti-CD3 $\varepsilon$ antibody by antiCD3 $\varepsilon$ antibody used for staining, we used magnetically sorted $\mathrm{CD}^{+}$cells containing approximately $95 \% \mathrm{CD}^{+} \mathrm{CD} 4^{+} \mathrm{Th}$ cells (cf. a representative dot plot shown in Figure 4A). IL-5 and IL-13 proteins were predominantly produced by $\mathrm{CD} 62 \mathrm{~L}^{\text {lo }} \mathrm{CXCR} 3^{\text {lo }}$ pathogenic Th2 cells after TCR stimulation in both WT and Exph5-KO mice, and secretion of neither cytokine was altered by exophilin- 5 deficiency (Figure 4B and Supplemental Figure 2B). Consistently, the expression levels of GATA3, the master transcription factor for induction of Th2 cytokines (26), were not affected by exophilin- 5 deficiency (Supplemental Figure 2, C and D). These findings indicate that the net IL-5 and IL-13 production by total CD $4^{+}$Th cells after TCR stimulation discussed hereafter mostly reflects its production by pathogenic Th2 cells.

It has been shown that pathogenic Th2 cells express the IL-33 receptor (IL-33R) (also known as interleukin 1 receptor like1 [IL1RL1] and ST2), and that IL-33 is vital for the induction and activation of pathogenic Th2 cells (27). In fact, among 4 fractions of splenic memory $\mathrm{CD} 4^{+} \mathrm{T}$ cells obtained from OVA-sensitized WT mice, mRNA levels of Il1rl1, which encodes IL-33R, were the highest in pathogenic Th2 cells (Figure 5A). It is also reported that IL-33 enhances IL- 5 and IL-13 production by TCR-activated CD $4^{+} \mathrm{T}$ cells $(28,29)$. Because IL-33 levels released into alveolar spaces increased along 


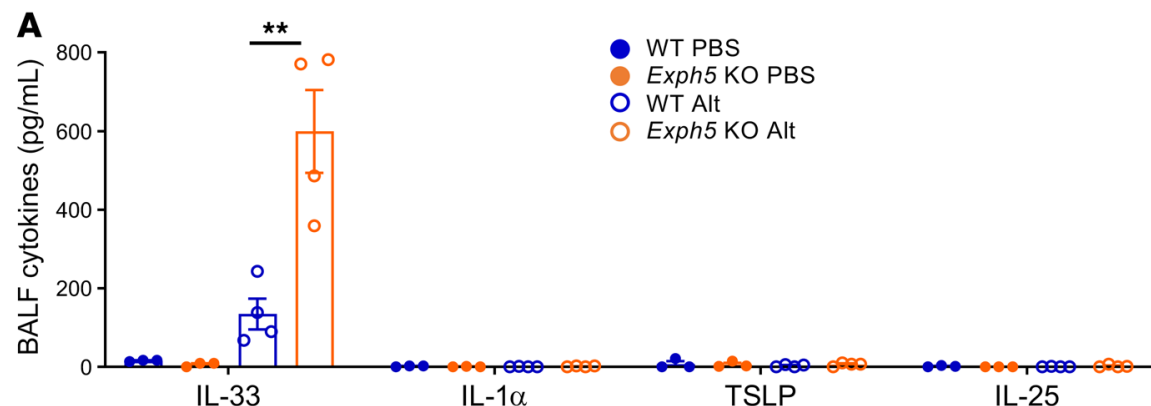

B IL-33 levels in OVA-induced allergic airway inflammation
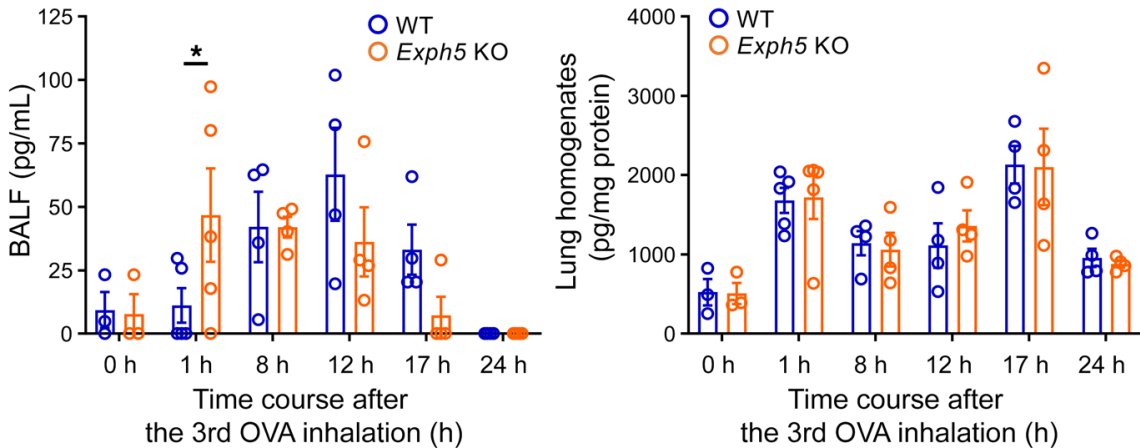

the 3rd OVA inhalation (h)

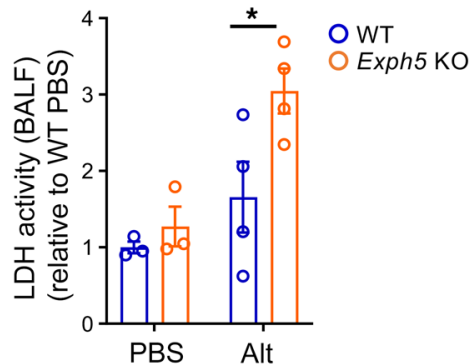

C IL-33 levels in BM chimera mice

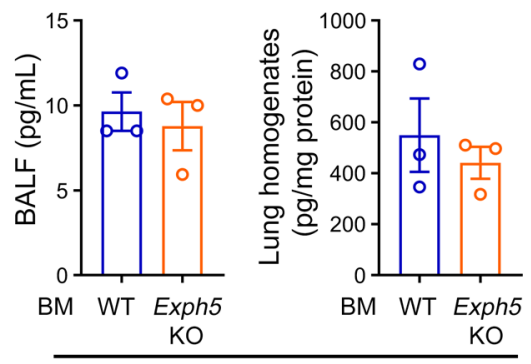

Recipient: WT

Figure 3. IL-33 secretion is enhanced in the lungs of Exph5-KO mice. (A) Levels of epithelial cell-derived cytokines and LDH in BALF of mice obtained 30 minutes after intratracheal injections of PBS or $10 \mu \mathrm{g}$ of Alternaria extract (Alt). LDH levels were normalized to the averages of those in PBS-treated WT mice. ${ }^{*} P<0.05 ;{ }^{* *} P<0.01$ by unpaired $t$ test. (B) IL-33 levels in the lungs of mice after the final OVA aerosol challenge. At the indicated time point after the third OVA aerosol challenge, BALF and lung tissues were collected from OVA-sensitized mice, and IL-33 concentrations in BALF and lung homogenates were determined by ELISA. (C) IL-33 levels in the lungs of mice at 1 hour after the final OVA aerosol challenge. ${ }^{*} P<0.05$ by paired $t$ test. Data were obtained from $n=3-5$ mice gathered from 2 to 3 independent experiments.

with increased IL-33 production in OVA-challenged lungs (Figure 3B), we investigated whether exophilin-5 deficiency enhanced IL-5 and IL-13 production by pathogenic Th2 cells in response to IL-33. Splenic CD $4^{+} \mathrm{T}$ cells derived from mice transplanted with Exph5KO BM produced much higher levels of IL-5 and IL- 13 in response to IL-33 under TCR stimulation compared with those cells from mice transplanted with WT BM (Figure 5B and Supplemental Figure $2 \mathrm{E}$ ). By contrast, exophilin- 5 deficiency in $\mathrm{CD} 4^{+} \mathrm{T}$ cells did not affect production of either IL-4 or IFN- $\gamma$ in response to the same stimulation (Supplemental Figure 2F). It was recently reported that tissue-resident memory $\mathrm{T}$ cells reside in the lungs (30). Therefore, we isolated lung $\mathrm{CD}^{+} \mathrm{T}$ cells from mice transplanted with WT BM or Exph5-KO BM, and examined responsiveness to IL-33 in those cells. We found that, similar to splenic $\mathrm{CD} 4^{+} \mathrm{T}$ cells, exophilin-5-deficient lung CD4 ${ }^{+} \mathrm{T}$ cells produced higher levels of IL-5 and IL-13 in response to TCR and IL-33 stimulation compared with WT lung $\mathrm{CD}^{+} \mathrm{T}$ cells (Supplemental Figure $2 \mathrm{G}$ ). Because similar phenotypes were observed in both splenic and lung-resident $\mathrm{CD}^{+}$ $\mathrm{T}$ cells, and the numbers of lung-resident $\mathrm{CD} 4^{+} \mathrm{T}$ cells were limited (5\%-10\% of splenic CD $4^{+} \mathrm{T}$ cells), we used splenic CD $4^{+} \mathrm{T}$ cells for further analyses. These results indicate that exophilin- 5 deficiency in pathogenic Th2 cells enhances responsiveness to IL-33, which results in increased IL-5 and IL-13 production by these cells in the presence of both TCR and IL-33 stimulation. Consistent with this idea, the exacerbation of eosinophilic lung inflammation observed in WT recipient mice transplanted with Exph5-KO BM (Figure 2B) or with splenic $\mathrm{CD} 4^{+}$Th cells was nullified in $\mathrm{Il33- \textrm {KO }}$ recipient mice (Figure 5, C and D), indicating that enhanced allergic lung inflammation induced by exophilin-5 deficiency in BM cells and $\mathrm{CD} 4^{+} \mathrm{T}$ cells was dependent on IL-33.

Although exophilin-5 was expressed in CD11 $\mathrm{c}^{+}$splenic DCs at a low level (Figure 4A), exophilin-5 deficiency did not affect these DCs' ability to polarize Th cells into Th1 cells or Th2 cells (Supplemental Figure 3A). Furthermore, exophilin-5 deficiency did not change IL-5/IL-13 production in response to IL-33 by either lung CD $45^{+}$leukocytes (Supplemental Figure 3B) or lung type-2 innate lymphoid cells (ILC2s) (Supplemental Figure 3C), suggesting that it did not enhance IL-33-stimulated, antigen-independent IL-5 or IL-13 production in the lungs. Taken together, these results show that exophilin-5 deficiency seems to augment allergic lung inflammation by enhancing IL-33 responsiveness in IL-5/IL-13-producing pathogenic Th2 cells.

Enhanced allergic inflammation in exophilin-5-deficient mice is dependent on IL-33. To demonstrate IL-33 dependency of the net exacerbated allergic immune responses in exophilin-5-deficient mice, we generated Exph5 and Il33 double KO (DKO) mice. We found that enhanced allergic airway inflammation in Exph5-KO mice was completely inhibited in Exph5 and Il33 DKO mice in an OVA-induced mouse model of asthma (Figure 6A). We also confirmed similar results in a house dust mite-driven mouse model of asthma (Figure 6B). These results indicate that phenotypes observed in Exph5-KO mice were almost completely dependent on IL-33. 
A Th $\left(\mathrm{CD}^{+} \mathrm{CD}^{+}\right)$

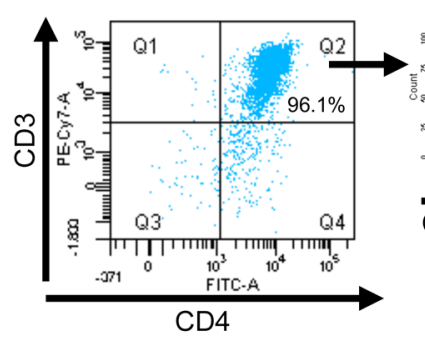

$\operatorname{Tm}\left(C D 44^{\text {hi }}\right)$

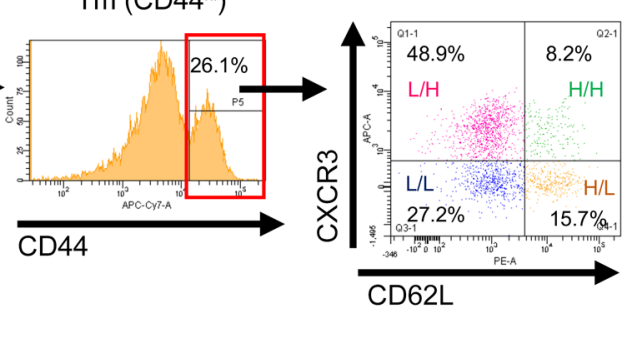

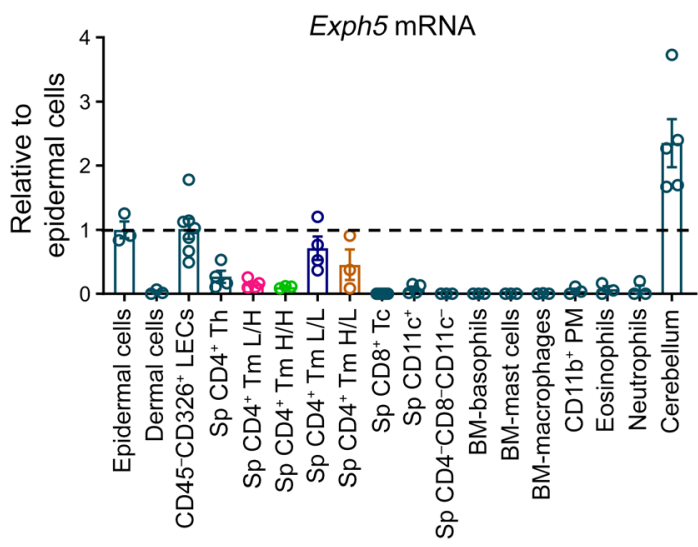
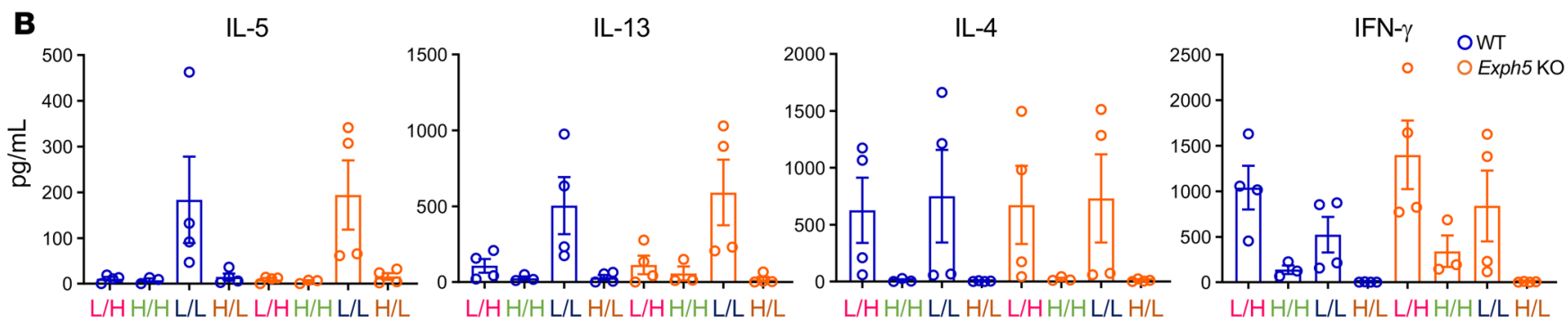

Figure 4. Exophilin-5 is highly expressed in pathogenic Th2 cells. (A) Exph5 mRNA levels in cell fractions. Exph5 mRNA levels were normalized to the average values in epidermal cells. Left: Sorting strategy of 4 populations divided by levels of CXCR3 and CD62L in CD $3^{+} C D 4^{+} C D 44^{\text {hi }}$ splenic memory T cells. Right: Exph5 mRNA expression in each cell fraction ( $n=3-7)$. Sp, splenic; PM, peritoneal macrophages; cerebellum, positive control; L/L, CD62 $\mathrm{L}^{\circ} \mathrm{CXCR} 3^{\text {lo; }}$ $\mathrm{L} / \mathrm{H}, \mathrm{CD} 62 \mathrm{~L}^{\text {lo }} \mathrm{CXCR} 3^{\text {hi }} ; \mathrm{H} / \mathrm{H}, \mathrm{CD} 62 \mathrm{~L}^{\text {hi }} \mathrm{CXCR} 3^{\text {hi }} ; \mathrm{H} / \mathrm{L}, \mathrm{CD} \mathrm{LL}^{\text {hic }} \mathrm{CXCR} 3^{\text {lo }}$. (B) Profile of cytokine secretion upon TCR stimulation by 4 fractions of splenic memory Th cells obtained from nonsensitized WT or Exph5-KO mice. Sorted cells (refer to the dot plot in A) were stimulated with anti-CD3 $\varepsilon$ monoclonal Ab (CD3) for 2 days ( $n=3$ for $\mathrm{H} / \mathrm{H}$ and $n=4$ independent experiments for others). Cytokine levels in the supernatants were then determined by ELISA.

Exophilin-5 deficiency increases the specialized IL-5/IL-13 producers, IL-33R-expressing pathogenic Th2 cells. Because IL-33 signals through its receptor IL-33R, we next investigated the effect of exophilin-5 deficiency on IL-33R expression on pathogenic Th2 cells. We isolated splenic $\mathrm{CD}^{+} \mathrm{T}$ cells from BM-chimeric mice (recipient, WT mice; donor BM, WT mice or Exph5-KO mice), and examined expression levels of cell surface IL-33R on $\mathrm{CD} 44^{\text {hi }} \mathrm{CD} 62 \mathrm{~L}^{\text {lo }} \mathrm{CXCR} 3^{\text {lo }}$ pathogenic Th 2 cells. The percentages of $\mathrm{CD} 44^{\text {hi }}{ }^{\mathrm{CD}} 62 \mathrm{~L}^{\text {lo }} \mathrm{CXCR} 3^{\text {lo }}$ pathogenic Th 2 cells in $\mathrm{CD} 4{ }^{+} \mathrm{T}$ cells were similar between mice transplanted with WT BM and those transplanted with Exph5-KO BM (Figure 7A). Upon TCR stimulation, the percentages of IL-33R $\mathrm{R}^{+}$pathogenic $\mathrm{Th} 2$ cells were significantly increased only in mice transplanted with Exph5-KO-derived BM (Figure 7B), without an increase in IL-33R mRNA levels (Supplemental Figure 4A), suggesting that this increase in IL-33R expression is regulated at a step beyond transcription (e.g., translation). Such an increase in IL-33R ${ }^{+}$cells was not observed in the CD $44^{\text {hi }}$ CD62 $\mathrm{L}^{\text {hi }} \mathrm{CXCR} 3^{\text {lo }} \mathrm{Th}$ cell fraction, the other fraction with high exophilin-5 expression (Supplemental Figure 4B). IL-33R is encoded by the Il1rl1 gene from which soluble ST2, a splicing variant short isoform of IL-33R without the transmembrane motif that can function as a decoy receptor for IL-33 to decrease IL-33 binding to cell surface IL-33R, is also generated (31). We also examined the effect of exophilin-5 deficiency on soluble ST2 production, and found that exophilin- 5 deficiency did not affect production by $\mathrm{CD} 4^{+} \mathrm{T}$ cells, pathogenic Th2 cells, or in the whole body, of soluble
ST2 proteins (Supplemental Figure 4C), suggesting that enhanced IL-33 signaling in exophilin-5-deficient $\mathrm{CD} 4^{+} \mathrm{T}$ cells was not mediated by a decrease in soluble ST2 production. It should be noted that in contrast to IL-33R $\mathrm{R}^{-}$pathogenic Th2 cells, IL-33R $\mathrm{R}^{+}$pathogenic Th2 cells selectively produced IL-5 and IL-13, but neither IL-4 nor IFN- $\gamma$, in response to TCR stimulation (Figure 7C), indicating that these IL- $33 \mathrm{R}^{+}$cells are the specialized IL- 5 and IL- 13 producers. It is worth mentioning that the numbers of $\mathrm{CD} 4^{+} \mathrm{T}$ cells used in Figure 7C was just one-tenth of those used in the other experiments. In addition, we also clarified that Exph5 mRNA was significantly enriched in pathogenic Th2 cells expressing a high level of Illrl1 mRNA (Figure 7D). These findings possibly explain why a relatively small increase in the number of $\mathrm{IL}^{-} 33 \mathrm{R}^{+}$pathogenic $\mathrm{Th} 2$ cells (Figure 7B) significantly enhances IL-5 and IL-13 production in exophilin-5-deficient $\mathrm{CD}^{+} \mathrm{T}$ cells after simultaneous TCR and IL-33 stimulation (Figure 5B). To expand our findings to human $\mathrm{CD}^{+} \mathrm{T}$ cells, we isolated $\mathrm{CD} 4^{+} \mathrm{CCR} 4^{+}$cells from human peripheral blood as a memory-type Th2-enriched cell fraction $(20,32)$, and examined EXPH5 and IL1RL1 mRNA levels in each sample. We found that EXPH5 mRNA levels positively correlated with IL-33R-encoding IL1RL1 mRNA levels in these cells (Figure 7E), implying a functional connection between IL-33R and exophilin-5 also in human Th2 cells.

Exophilin-5 deficiency enhances $I L-33$ responsiveness in $I L-5$ - and IL-13-producing pathogenic Th2 cells through augmentation of the PI3K/Akt/mTOR pathway. We next sought to clarify the mech- 
A

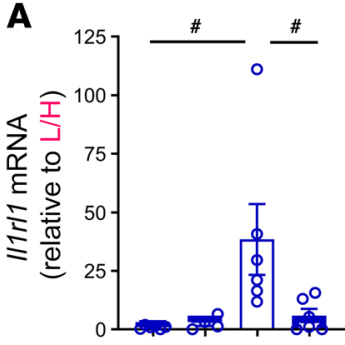

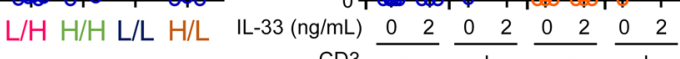

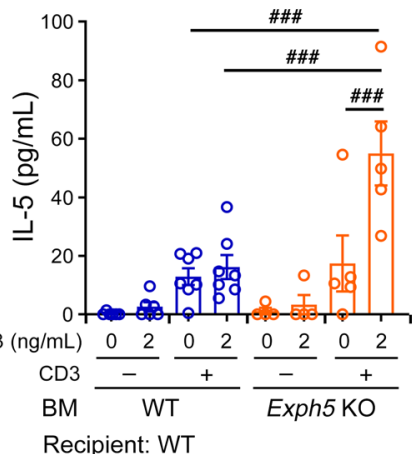

Recipient: WT

B

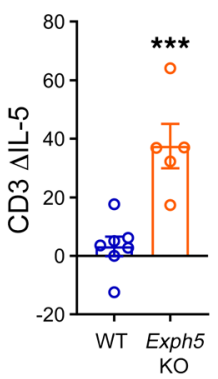

$\mathrm{KO}$

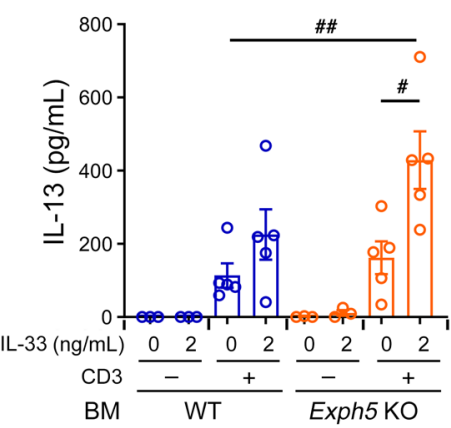

Recipient: WT
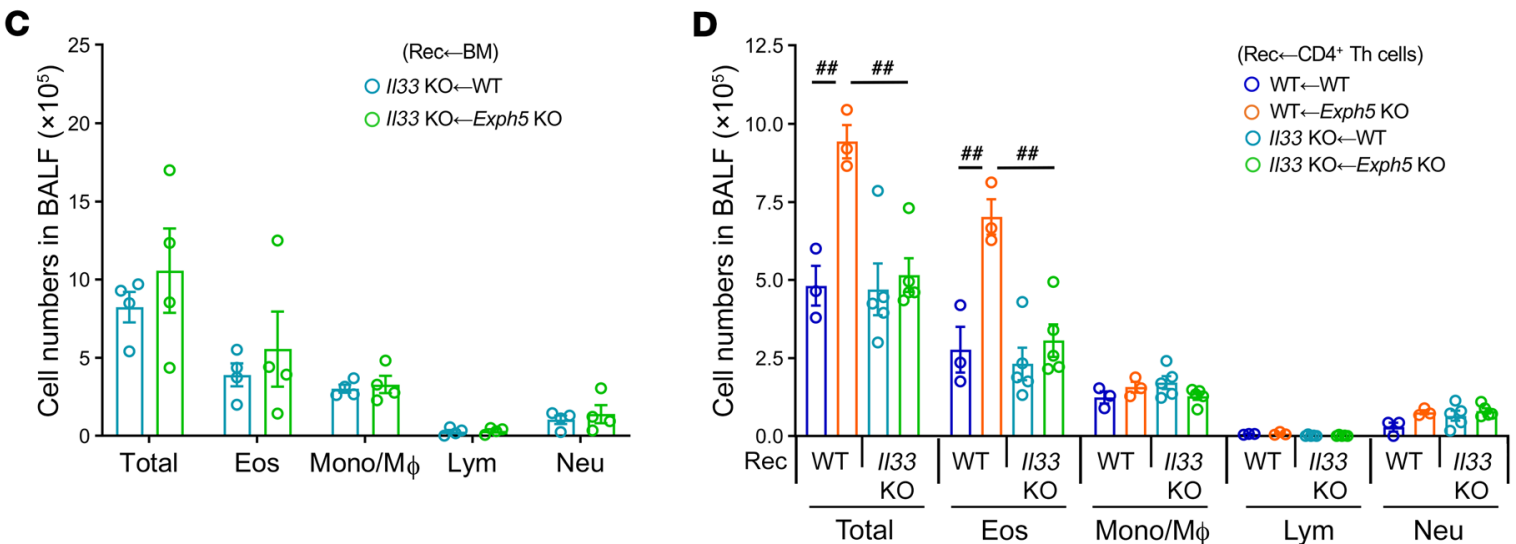

Figure 5. Exophilin-5 deficiency enhances IL-33-mediated IL-5 and IL-13 production by pathogenic Th2 cells. (A) IL1RL1 mRNA levels in 4 fractions of splenic CD44 ${ }^{\text {hi }}$ memory Th cells ( $n=4-6$ gathered from 4 independent experiments). L/L, CD62 $L^{\text {lo } C X C R ~} 3^{10} ; L / H, C D 62 L^{10} C X C R 3^{\text {hi }} ; H / H, C D 62 L^{\text {hi }} C X C R 3^{\text {hi }}$; $\mathrm{H} / \mathrm{L}, \mathrm{CD} 62 \mathrm{~L}^{\text {hicXCR }}{ }^{\text {lo }}$. (B) IL-5 and IL-13 production upon TCR and IL-33 stimulation by splenic CD4+ ${ }^{+}$cells obtained from OVA-sensitized mice. CD4 ${ }^{+}$T cells obtained from mice transplanted with WT BM or with Exph5-KO BM were cultured with and without anti-CD3\& Ab (CD3) with and without IL-33 (2 ng/mL) for 2 days. Levels of cytokines in the supernatants were then determined by ELISA. $\Delta \mathrm{IL}-5$ and $\Delta \mathrm{IL}-13$ indicate the increase in cytokine production induced by addition of IL-33. Data were obtained from mice $(n=3-7)$ gathered from at least 2 independent experiments. (C and $\mathbf{D})$ The role of IL-33 in exacerbated allergic lung inflammation induced by exophilin-5 deficiency. (C) BALF cell numbers after 3-day OVA airway challenges in I/33-KO mice transplanted with either WT BM or Exph5-KO BM cells ( $n=4$ mice). (D) BALF cell numbers after 3-day OVA airway challenges in WT or II33-KO mice that received CD4 ${ }^{+}$T cells obtained from WT or Exph5-KO mice ( $n=3-5$ mice per group obtained from 2 independent experiments). ${ }^{*} P<0.05 ;{ }^{* *} P<0.001$ by unpaired $t$ test. $\# P<0.05 ; \# P<0.01 ; \# \# P<0.001$ by 1-way ANOVA with Tukey's post hoc test.

anism by which exophilin-5 deficiency increases expression of IL-33R in pathogenic Th2 cells. Because previous studies have suggested that IL-33R expression is induced by the phosphoinositide 3-kinase (PI3K)/Akt/mTOR pathway $(33,34)$, we first investigated whether this pathway was augmented in exophilin-5-deficient pathogenic Th2 cells. We observed that the percentage of pathogenic Th2 cells expressing high levels of phosphorylated Akt (p-Akt), a downstream product of PI3K, rapidly increased after 15 minutes and 30 minutes of TCR stimulation in exophilin-5-deficient $\mathrm{CD} 4^{+} \mathrm{Th}$ cells (Figure 8A and Supplemental Figure 5A). In WT CD $4^{+}$Th cells, no such increase was observed in p-Akt ${ }^{\mathrm{hi}}$ cells at these early time points. These results suggest that exophilin-5 deficiency somehow enhances the activity of the PI3K/Akt/ mTOR pathway. Consistent with this observation, the PI3K inhibitor wortmannin significantly decreased the number of IL-33R ${ }^{+}$ pathogenic Th2 cells (Figure 8B) and IL-5 and IL-13 production in response to IL-33 (Figure 8C and Supplemental Figure 5B) only in exophilin-5-deficient $\mathrm{CD}^{+}$Th cells. The mTOR inhibitor rapamycin also significantly suppressed IL-5 and IL-13 production in response to IL-33 in exophilin-5-deficient CD4 $4^{+}$Th cells (Figure $8 \mathrm{D}$ and Supplemental Figure 5C). mTOR is well known to increase protein synthesis by enhancing protein translation (35). Considering our results that exophilin-5 deficiency did not increase IL-33R mRNA levels (Supplemental Figure 4A), the increase in IL-33Rexpressing cells in exophilin-5-deficient pathogenic Th2 cells is possibly mediated by enhanced protein translation. Taken together, these results suggest that exophilin-5 deficiency enhances IL-33 sensitivity in pathogenic Th2 cells via augmentation of the PI3K/Akt/mTOR pathway.

Exophilin-5 deficiency enhances the PI3K/Akt/mTOR pathway by changing the localization of NADPH oxidase in pathogenic Th2 cells. Next, we investigated the mechanism by which exophilin-5 deficiency enhances the activity of the PI3K/Akt/mTOR pathway upon TCR stimulation. Among many types of vesicles whose intracellular trafficking is reported to be regulated by Rab27, we focused on those harboring Nox2, a phagocyte-type NADPH oxidase mainly expressed in macrophages and neutrophils. Rab27a is known to regulate intracellular trafficking of Nox2-containing vesicles upon stimulation in these cells $(9,10)$. It is also reported that increased intracellular ROS production regulated by NADPH oxidases activates the PI3K/Akt/mTOR pathway $(36,37)$. Furthermore, among 6 different types of NADPH oxidases, Nox 2 is 
A

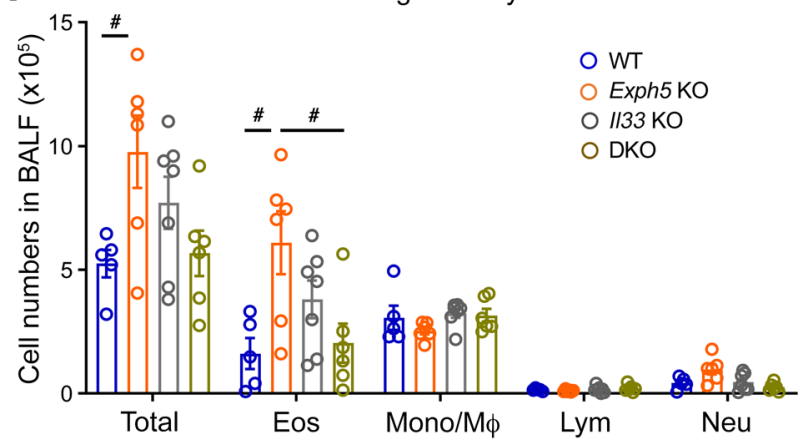

B HDM-induced allergic airway inflammation

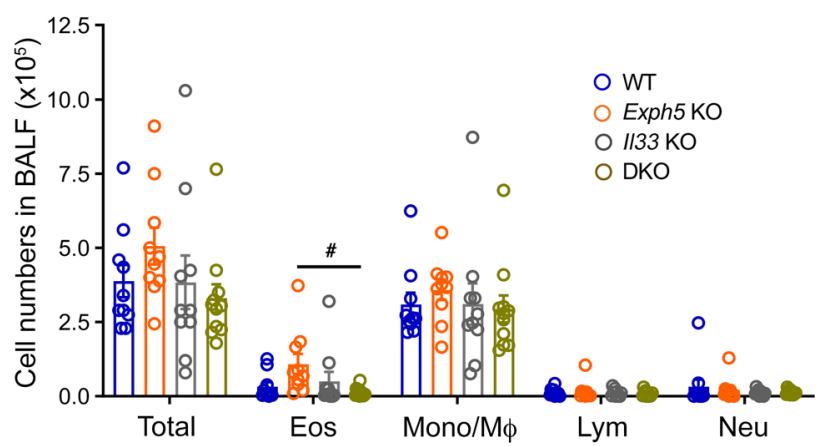

OVA-induced allergic airway inflammation

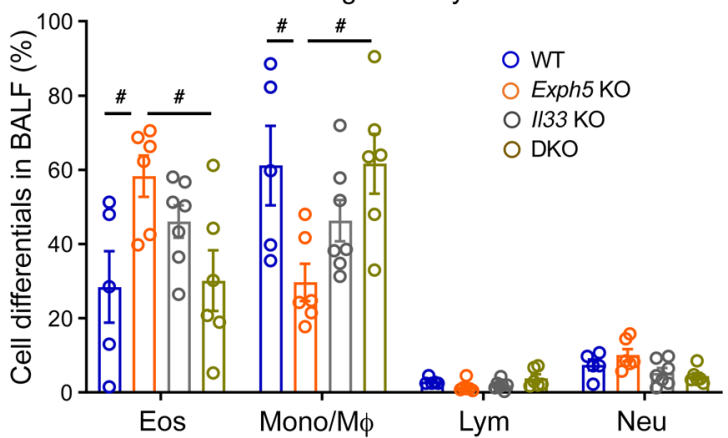

HDM-induced allergic airway inflammation

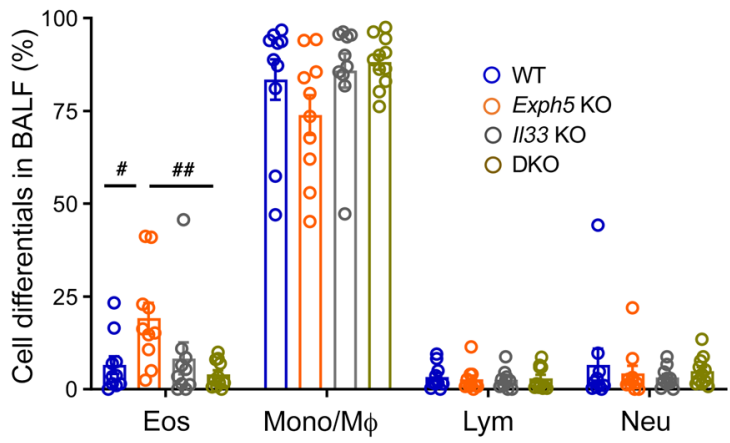

Figure 6. Exacerbation of allergic lung inflammation in Exph5-KO mice is completely inhibited by lack of IL-33. Cell numbers and differentials in BALF after 3-day $3 \%$ OVA inhalation (A) or after 7 intranasal house dust mite (HDM) injections (B). Data were obtained from $n=5-7$ mice combined from 2 independent experiments in $\mathbf{A}$ and from $n=10-11$ mice combined from 5 independent experiments in $\mathbf{B}$. ${ }^{\#} P<0.05 ;{ }^{\#} P<0.01$ by 1-way ANOVA with Tukey's post hoc test.

expressed in $\mathrm{CD}^{+} \mathrm{T}$ cells (38) and is responsible for extracellular superoxide release by $\mathrm{CD} 4^{+} \mathrm{T}$ cells upon TCR stimulation (39). In fact, the levels of Nox2-encoding Cybb mRNA in pathogenic Th2 cells were much higher than those in total $\mathrm{CD} 4^{+}$Th cells (Figure 9A) or 3 other fractions of splenic memory-type Th cells, and were not affected by exophilin-5 deficiency (Figure 9B). We found that Nox2 bound Rab27a, the major Rab27 isotype expressed in T cells $(19,40)$, and formed a protein complex with exophilin-5 in the presence of Rab27a in HEK293A cells (Figure 9C). Furthermore, Nox2 was translocated from the cell interior to the plasma membrane in WT pathogenic Th2 cells upon stimulation with PMA and ionomycin, which has been used to mimic TCR stimulation, whereas this was not the case in exophilin-5-deficient cells; the percentages of cells with complete loss of cytoplasmic Nox2 staining upon stimulation were $26.5 \%$ in WT cells and $4.2 \%$ in Exph5-KO cells (approximately 80 cells per mice; Figure 9D). Consistent with this finding, superoxide release after the same stimulation was significantly decreased in Exph5-KO pathogenic Th2 cells (Figure 9E). Intracellular ROS after stimulation with PMA/ionomycin (Figure 9F) or TCR stimulation (Figure 9G) was reciprocally increased in exophilin-5-deficient pathogenic Th2 cells. These results strongly suggest that exophilin-5 deficiency inhibits intracellular movement of Nox 2 to the plasma membrane and that Nox2 residing in the cytosol produces ROS intracellularly upon stimulation in pathogenic Th2 cells. Although the lack of a specific antibody suitable for immunostaining mouse exophilin-5 and the extremely inefficient induction of exogenous exophilin-5 due to its large size prevented us from examining the association of exophilin-5 with Rab27a- or Nox2-containing vesicles in T cells, we found that exogenous Nox2 colocalized with Rab27a in CD4 ${ }^{+}$ $\mathrm{T}$ cells (Supplemental Figure 6A). Similarly to exophilin-5-deficient cells, $\mathrm{CD} 4^{+} \mathrm{T}$ cells from Rab27a-deficient ashen mice tended to enhance IL-5 production in response to IL-33 stimulation and to suppress extracellular superoxide secretion after PMA and ionomycin stimulation (Supplemental Figure 6, B and C). Although we could not directly clarify their interaction in $\mathrm{T}$ cells due to technical limitations described above, these findings suggest that Rab27a and exophilin-5 cooperatively regulate the trafficking of Nox2-containing vesicles in $\mathrm{CD} 4^{+} \mathrm{T}$ cells.

To establish the role of the NADPH oxidase-mediated signaling pathway in IL-33-dependent IL-5 and IL-13 production, we investigated the effect of the NADPH oxidase inhibitor, diphenyleneiodonium (DPI), on pathogenic Th2 cells. DPI significantly reduced the number of IL-33R $\mathrm{R}^{+}$cells (Figure 10A), and completely blocked the enhanced responsiveness to IL-33 in Exph5-KO mice (Figure 10B) and in mice transplanted with Exph5-KO BM (Figure 10C). These findings indicate that enhanced IL-33 sensitivity in pathogenic Th 2 cells induced by exophilin- 5 deficiency is caused by inhibition of the intracellular trafficking of Nox2-containing vesicles (Figure 10D).

\section{Discussion}

In the present study, we showed that deficiency of the Rab27 effector, exophilin-5, exacerbated allergic lung inflammation and that 


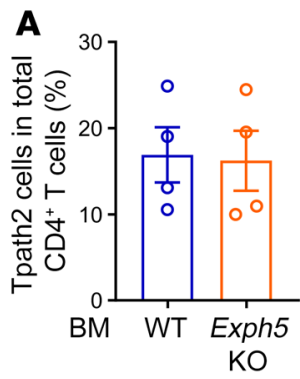

Recipient: WT
B

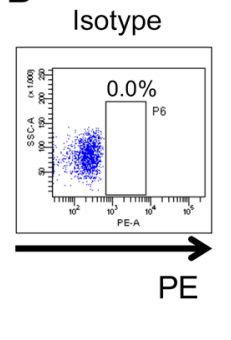

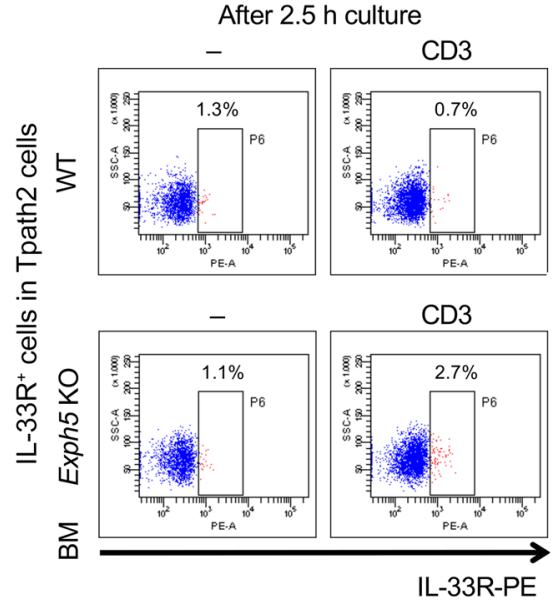
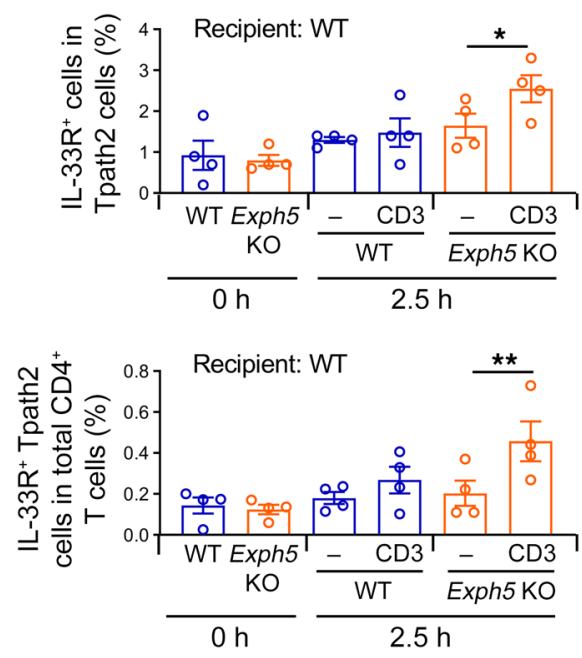

Cytokine secretion by pathogenic Th2 cells: IL-33R' vs IL-33R+

C

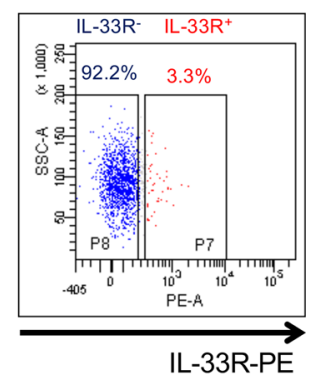

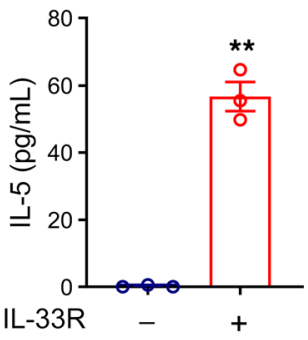

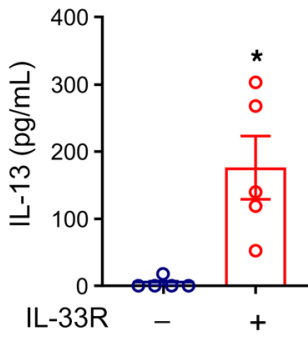

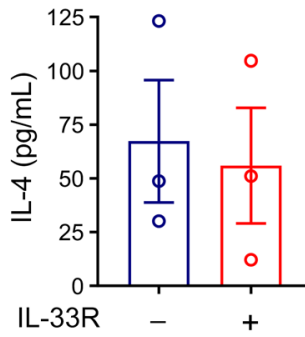

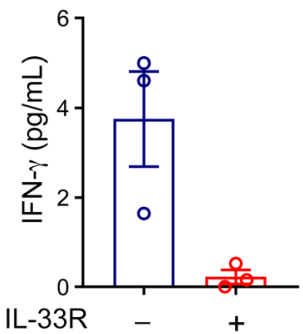

D

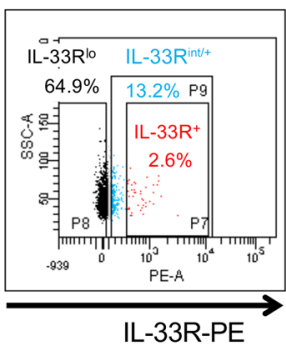

Gene expression of pathogenic Th2 cells: IL-33R ${ }^{\text {lo }}$ vs. IL-33R intt

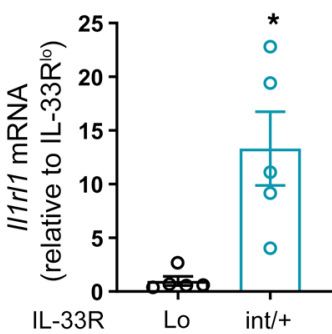

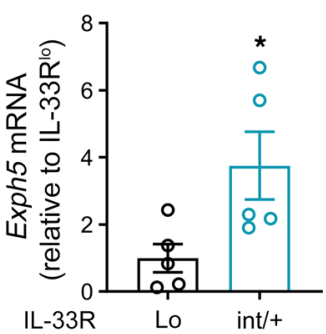

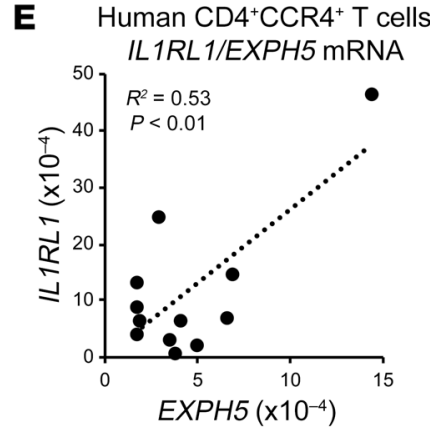

Figure 7. Exophilin-5 deficiency increases the specific IL-5/IL-13 producers, IL-33R-expressing cells, in pathogenic Th2 cells. (A) Percentages of pathogenic Th2 cells (Tpath2) in total CD4+ T cells before culture in each mouse. (B) Percentages of cells positive for cell surface IL-33R in pathogenic Th2 cells from each group. $C D 4^{+} T$ cells obtained from the indicated BM-chimeric mice were cultured with and without plate-bound anti-CD3\& Ab (CD3) for 2.5 hours. Then, cells were harvested and stained against cell surface molecules as described in Methods. Zero hours and 2.5 hours indicate before culture and after 2.5 hours of culture, respectively. A representative dot plot after 2.5 hours of culture is shown in B. (C) Cytokine profiles of IL-33R- versus IL-33R $\mathrm{R}^{+}$cells in pathogenic Th2 cells. Each fraction was sorted from splenic CD4+ Th cells of OVA-sensitized Exph5-KO mice, and were cultured at $1.5 \times 10^{4}$ cells/well (one-tenth of the cell number used in the other experiments) on anti-CD3\& Ab-coated wells for 2 days. Next, levels of typical Th2 and Th1 cytokines in the supernatants were

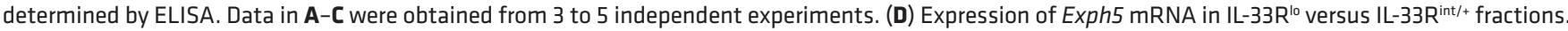
Each fraction was sorted from splenic CD4 ${ }^{+}$T cells of WT mice, and the levels of $1 / 1 r / 1$ mRNA and Exph5 mRNA in the 2 fractions were determined. Data were obtained from 5 independent experiments. (E) Pearson's correlation between IL1RL1 and EXPH5 mRNA expression in human Th2-enriched CD4+CCR4 ${ }^{+} T$ cells. Human CD4+CCR4 ${ }^{+} T$ cells, which contain memory-type Th2 cells, were isolated from human peripheral blood, and the mRNA levels relative to ACTB ( $\beta$-actin) were determined as described in Methods ( $n=12$ subjects). ${ }^{*} P<0.05 ;{ }^{* *} P<0.01$ by paired (B) or unpaired (C and D) $t$ test.

exophilin-5 regulates antigen-induced allergic immune responses by mediating responsiveness to IL-33 in IL-5/IL-13-producing pathogenic Th2 cells. This is the first study to our knowledge to describe this role of exophilin-5, a regulator of vesicle trafficking, in allergic immune responses.

We first hypothesized that exophilin-5 regulates allergic lung inflammation by controlling secretion of cytokines. Although exophilin-5-deficient lung epithelial cells indeed enhanced IL-33 release, possibly due to their fragility, exophilin-5-deficient hematopoietic immune cells did not show altered cytokine secretion. Given that exophilin-5 deficiency in hematopoietic cells completely phenocopied the exacerbated allergic lung inflammation in Exph5-KO mice, there could be other mechanisms in play. We found that exophilin-5 deficiency enhances IL-5 and IL-13 
A
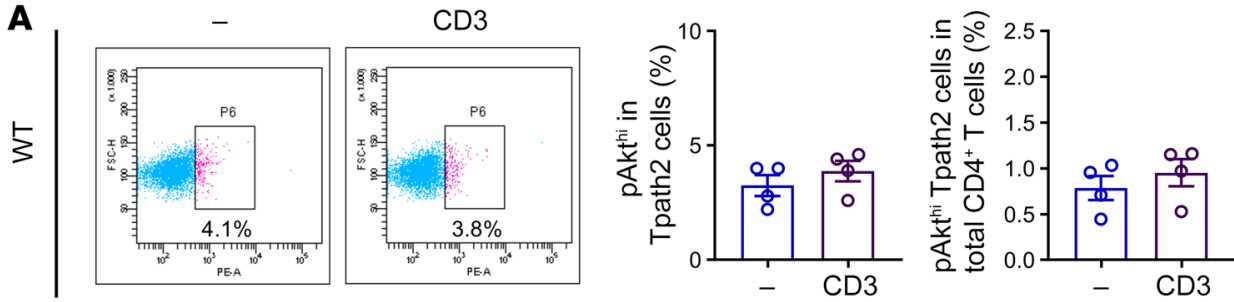

B
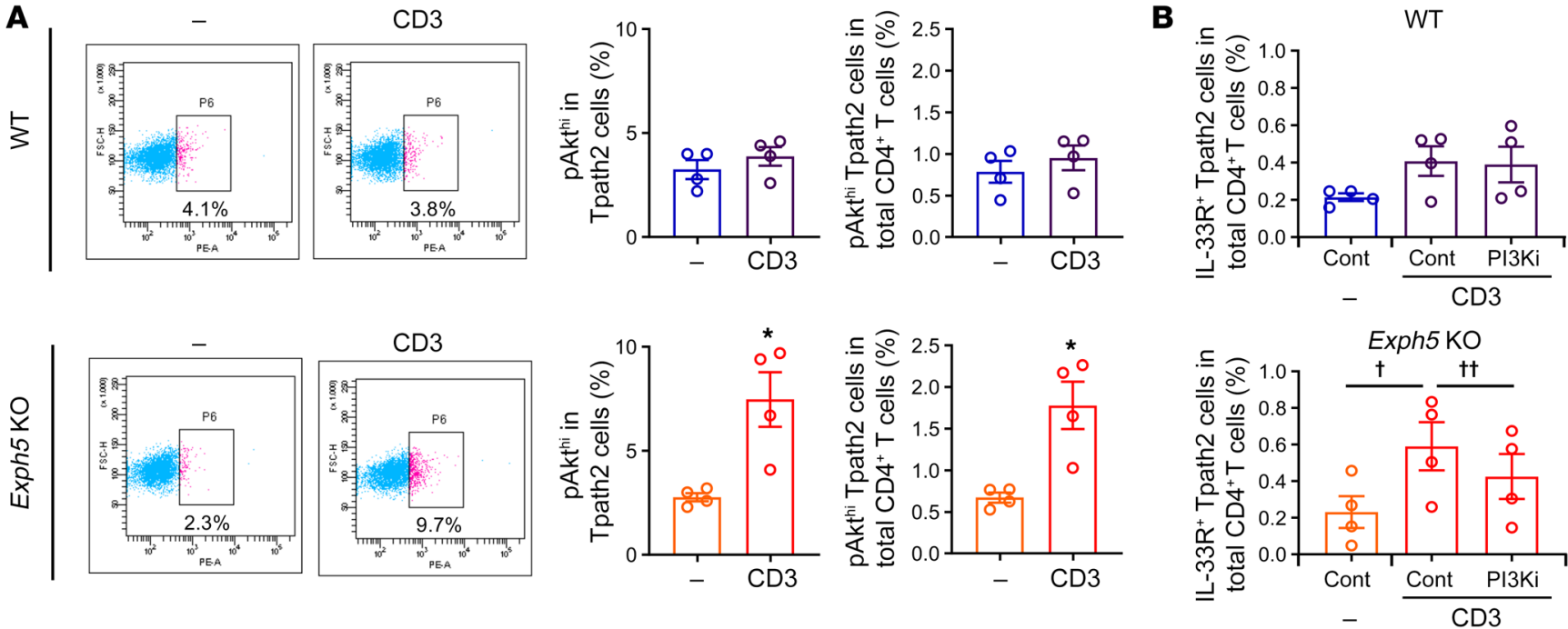
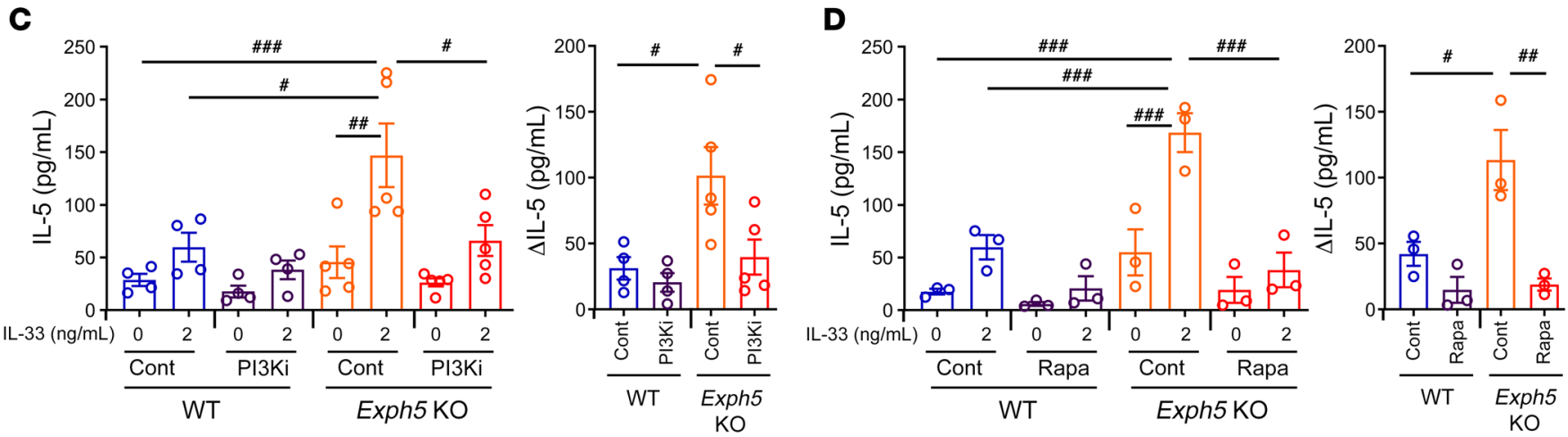

Figure 8. Enhanced IL-5 and IL-13 production in response to IL-33 stimulation by exophilin-5-deficient CD4 ${ }^{+}$cells is mediated by the PI3K/Alt/mTOR

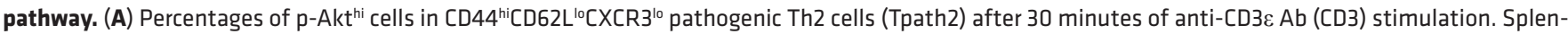
ic CD4+ $T$ cells obtained from OVA-sensitized WT mice or Exph5-KO mice were first stained for cell surface molecules, and were then cultured on anti-CD3E Ab-coated plates. After a 30-minute culture, cells were fixed, permeabilized, and stained with anti-p-Akt-PE Ab. (B) Effect of the PI3K inhibitor wortmannin (PI3Ki, $100 \mathrm{nM}$ ), on cell surface IL-33R expression. (C and D) Effects of wortmannin (C) and the mTOR inhibitor rapamycin (Rapa, $10 \mathrm{nM}$ ) (D) on IL-5 and IL-13 production by CD4+ $T$ cells. After a 10 -minute preincubation with and without the indicated inhibitors, $C D 4^{+} \mathrm{T}$ cells were cultured as described in Figure $5 \mathrm{~A}$ for 2 days. Cytokine levels in the supernatants were then measured by ELISA. $\triangle \mathrm{IL}-5$ indicates the increase in IL-5 production induced by addition of IL-33. CD4 ${ }^{+}$T cells were obtained from OVA-sensitized WT and Exph5-KO mice. Data were obtained from $n=4$ independent experiments in $\mathbf{A}$ and B. $n=$ 3-5 mice in $\mathbf{C}$ and $\mathbf{D}$ gathered from 2 to 3 independent experiments. ${ }^{*} P<0.05$ by unpaired $t$ test. ${ }^{\#} P<0.05$; $\# P<0.01$; $\# \# P<0.001$ by 1 -way ANOVA with Tukey's post hoc test. ${ }^{\dagger} P<0.05{ }^{\dagger+} P<0.01$ by repeated-measures ANOVA with Tukey's post hoc test.

production through augmentation of PI3K/Akt/mTOR signaling in pathogenic Th2 cells, and that Nox2, an NADPH oxidase, is the target for exophilin-5 in this context. NADPH oxidases are known to modulate kinase activities by altering intracellular ROS production $(36,37,41)$. Although Nox2 is reported to be expressed in $\mathrm{T}$ cells in which it mediates the release of extracellular superoxide upon TCR stimulation (39), its biological significance and the regulation of its intracellular trafficking in $\mathrm{T}$ cells is unknown. We showed that pathogenic Th2 cells express much higher levels of Nox 2 than do total CD4 $4^{+} \mathrm{T}$ cells and 3 other fractions of memory-type Th cells, that exophilin-5 binds Nox2 through Rab27a, and that exophilin-5 positively regulates the trafficking of Nox2 from the cytoplasm to the plasma membrane upon stimulation in pathogenic Th2 cells. Exophilin-5 deficiency inhibited the intracellular trafficking of Nox2 upon stimulation, which increased intracellular ROS production, the activity of the PI3K/Akt/mTOR pathway, IL-33R expression, and finally IL-5 and IL-13 production in response to IL-33 in pathogenic Th2 cells. Our findings suggest that Nox 2 in pathogenic Th2 cells relieves cell stress by releasing superoxide to the extracellular space to inhibit overactivation of the cells in response to additional external stimuli such as IL-33, and that exophilin- 5 functions as a brake on this IL-33-mediated overactivation of pathogenic Th2 cells.

Among Th2 cytokines, IL-5 and IL-13 are the most essential effector cytokines for the development of asthmatic characteristics such as eosinophil infiltration, mucus production, and airway hyperresponsiveness in the lungs. IL-5 is the strongest activator of eosinophils (42), while IL-13 enhances all features of asthma (43). In clinical settings, neutralizing antibodies against IL-5 or the IL-13 receptor have been shown to be effective when used in the treatment of patients with asthma (44-47). Although all Th2 cytokines were thought to be released by a type of single cell population called Th2 cells (48), Endo et al. recently reported that, among effector cytokine-producing CD $44^{\text {hi }}$ splenic memory T cells, IL-5 
A

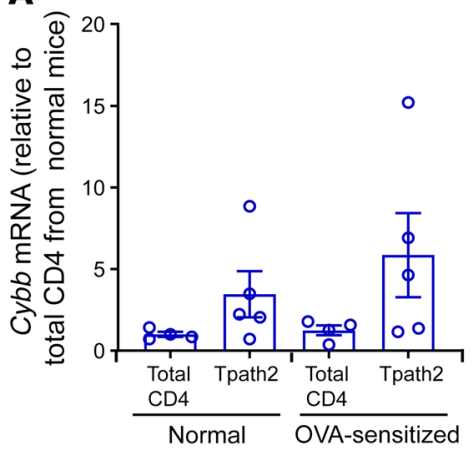

D

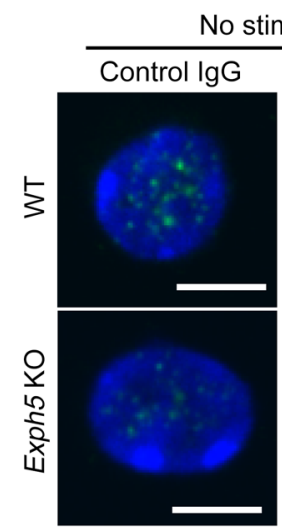

$\mathbf{F}$

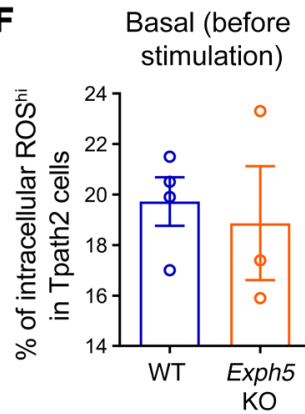

B

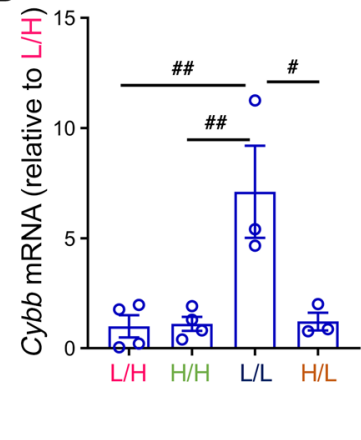

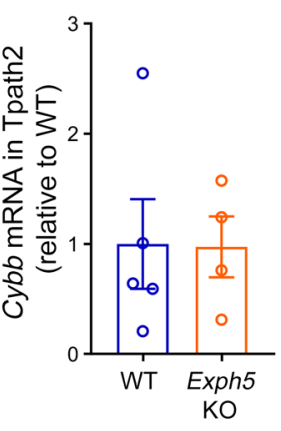

C

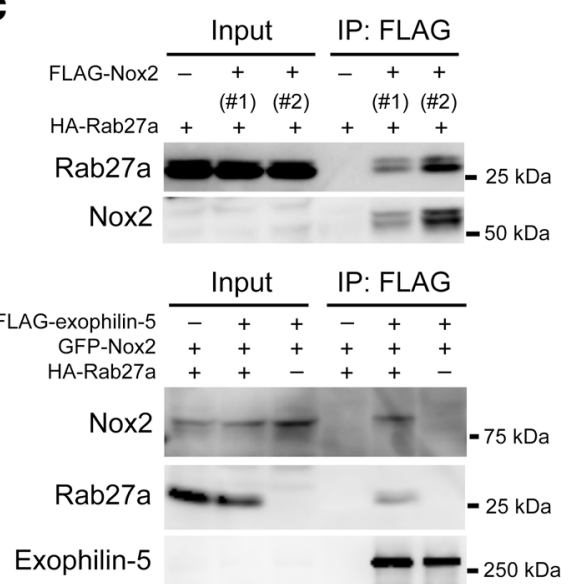

E

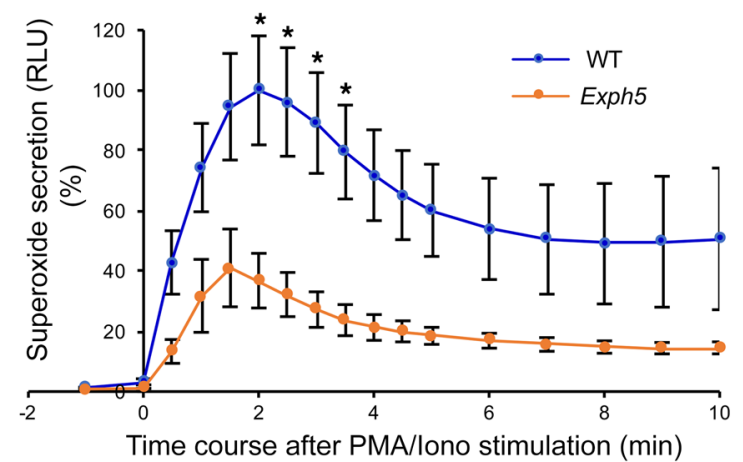

G

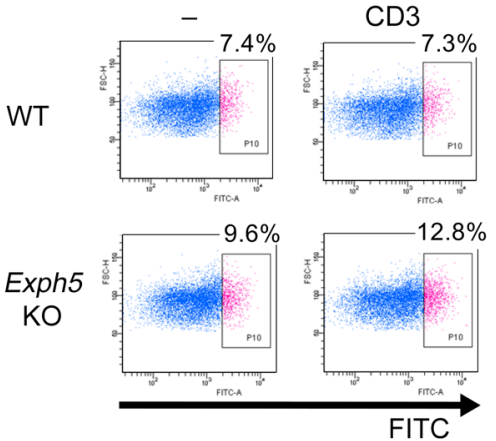

After 3 min PMA/lono

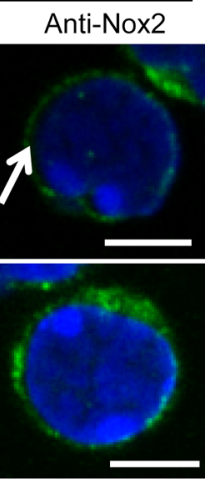

Exophilin-5

$-250 \mathrm{kDa}$

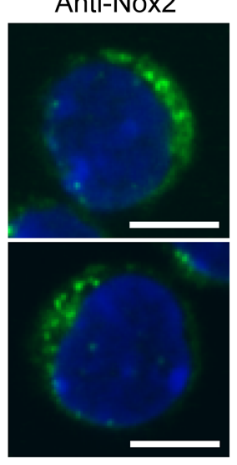

$\Delta$ Increase

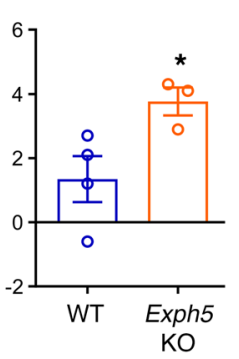

FITC

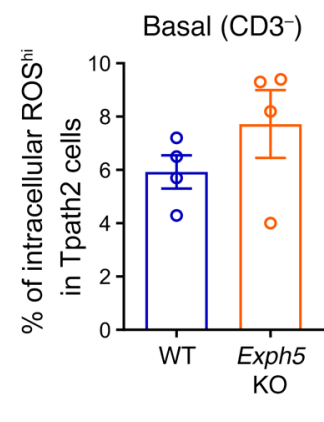

$\Delta$ Increase

Figure 9. Exophilin-5 deficiency inhibits intracellular trafficking of Nox2 upon stimulation in pathogenic Th2 cells. (A and B) Levels of Nox2-encoding Cybb mRNA in the indicated T cells (A, $n=4-5$ experiments), and in 4 fractions of CD44hiCD4+ $T$ cells (see Figure $3 A$ ) obtained from OVA-sensitized mice (B, $n=3-5$ experiments). ${ }^{P} P<0.05 ;{ }^{\# \# P}<0.01$ by 1 -way ANOVA with Tukey's post hoc test. Tpath2, pathogenic Th2 cells. L/L, CD62L $L^{10} \mathrm{CXCR} 3^{10} ; \mathrm{L} / \mathrm{H}, \mathrm{CD} 62 \mathrm{~L}^{10}$ $\mathrm{CXCR}^{\text {hi }} ; \mathrm{H} / \mathrm{H}, \mathrm{CD} \mathrm{L}^{\text {hi }} \mathrm{CXCR} 3^{\text {hi }} ; \mathrm{H} / \mathrm{L}, \mathrm{CD} 2 \mathrm{~L}^{\text {hi }} \mathrm{CXCR} 3^{\text {lo }}$. (C) Binding of Rab27a, Nox2, and exophilin-5. Transfection of plasmids into HEK293A cells, immunoprecipitation of cell lysates, and detection of the indicated proteins in immunoprecipitates were conducted as described in Methods. We used 2 clones of FLAG-tagged Nox2-expressing plasmids (upper), and then subcloned Cybb cDNA from plasmid number 2 into the pEGFP-C1 vector (lower). An immunoblot representative of 3 different experiments is shown. (D) Immunostaining of Nox2 in sorted pathogenic Th2 cells before and after a 3-minute stimulation with PMA plus ionomycin (PMA/lono). An image representative of 3 independent experiments is shown. The arrow in the WT cell indicates complete loss of cytoplasmic Nox2 staining. Scale bars: $5 \mu \mathrm{m}$. (E) Superoxide secretion by sorted pathogenic Th2 cells after PMA/lono stimulation. Data were obtained from a total of 4 mice ( $n=2$ from both nontreated and OVA-sensitized mice). (F and $\mathbf{G}$ ) Intracellular ROS production after stimulation. Pathogenic Th2 cells were sorted and cultured with and without PMA/lono stimulation (F) or anti-CD3E Ab (CD3) stimulation (G). (F) Percentages of ROS ${ }^{\text {hi }}$ population before stimulation (basal) and increases in them after 2.5 minutes of PMA/lono stimulation ( $\Delta$ increase) obtained from WT mice $(n=4)$ and Exph5-KO mice $(n$ = 3) gathered from 2 independent experiments are shown. (C) Left: A dot plot representative of 3 individual experiments is shown. Right: Percentages of

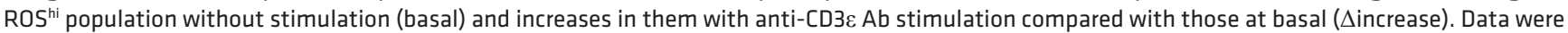
obtained from $n=4$ mice gathered from 3 independent experiments. ${ }^{*} P<0.05$ by unpaired $t$ test.

and IL-13 proteins are produced upon TCR stimulation selectively by a CD $62 \mathrm{~L}^{\text {lo }} \mathrm{CXCR} 3^{\text {lo }}$ pathogenic Th2 population, within which we found that exophilin-5 is highly expressed, while IL-4 is produced by multiple populations (24). They also reported that pathogenic
Th2 cells express IL-33R at a much higher level than do classical ex vivo-differentiated effector Th2 cells (27). We found that IL-5 and IL-13 producers represent only a minor subset (a few percentages) of murine splenic $\mathrm{CD} 62 \mathrm{~L}^{\mathrm{lo}} \mathrm{CXCR} 3^{\text {lo }}$ pathogenic $\mathrm{Th} 2$ cells, which 

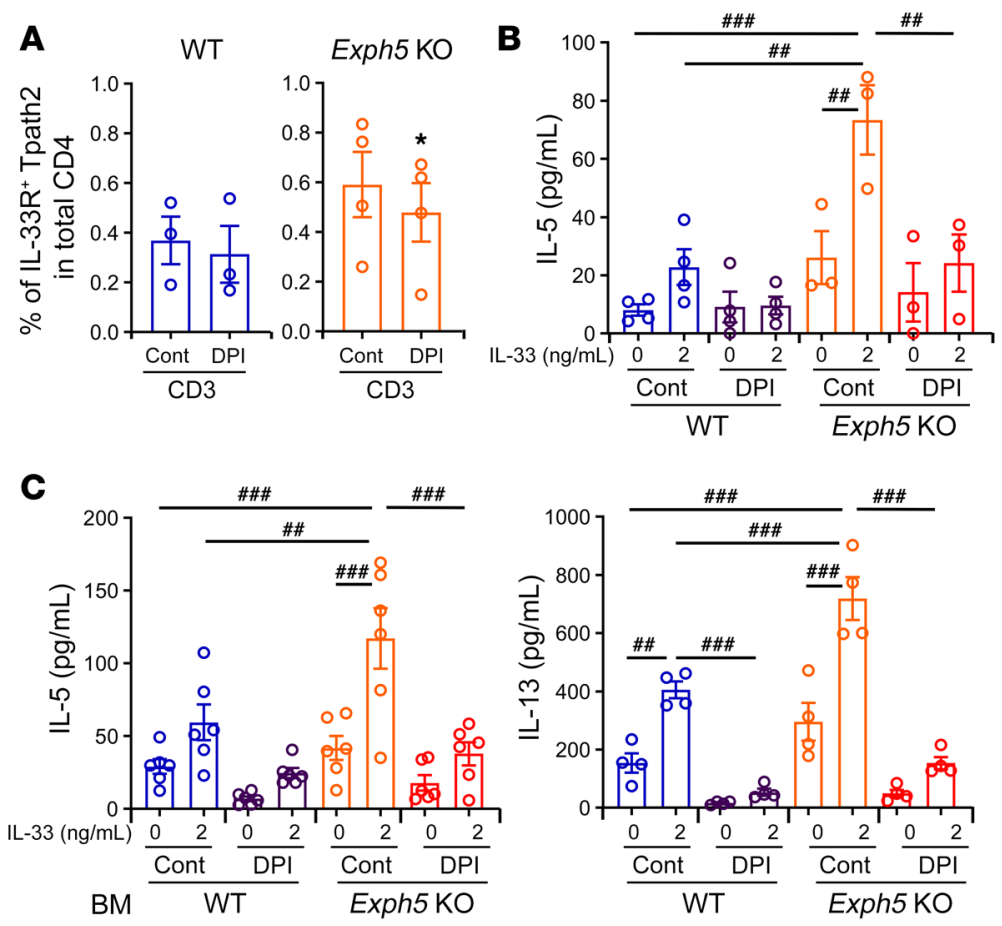

Recipient: WT

\section{D}

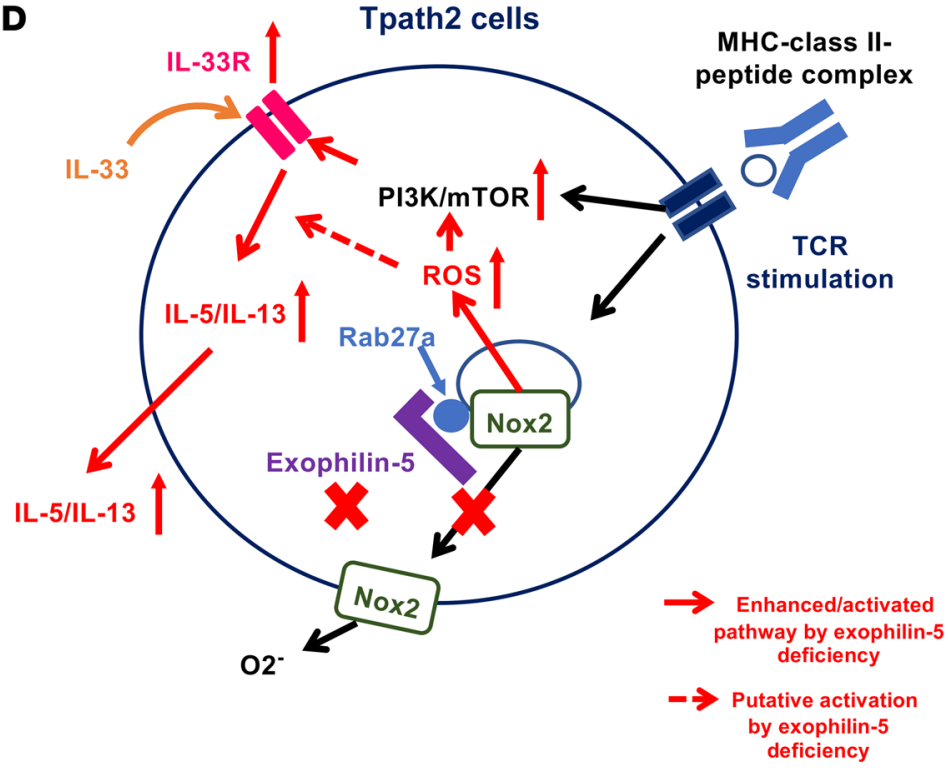

Figure 10. NAPDH oxidase inhibitor DPI significantly suppresses phenotypes in CD4 ${ }^{+} \mathrm{T}$ cells induced by exophilin- 5 deficiency. After a 10-minute preincubation with and without DPI (160 nM), cells were plated into anti-CD3ع Ab-coated wells to examine the effect of DPI on IL-33R expression (A) and on production of IL-5 and IL-13 (B and C). (A) Effect of DPI on IL-33R expression. After a 10-minute preincubation with and without DPI and 2.5 hours of culture in the presence of antiCD3\& Ab (CD3), cell surface IL-33R levels were determined. * $P$ $<0.05$ by paired $t$ test. Tpath2, pathogenic Th2 cells. (B and C) Effects of DPI on IL-33-mediated IL-5 production under TCR stimulation by $\mathrm{CD}^{+} \mathrm{T}$ cells obtained from OVA-sensitized WT and Exph5-KO mice (B), or on IL-33-mediated IL-5 and IL-13 production by $\mathrm{CD}^{+} \mathrm{T}$ cells from OVA-sensitized BM-chimeric mice (C). IL-5 levels in the supernatants after 2 -day culture were determined by ELISA as described in Figure $8, C$ and $D$. Data were obtained from $n=4-6$ mice from 2 to 4 independent experiments. ${ }^{\#} P<0.01$; $\# \# P<0.001$ by 1 -way ANOVA with Tukey's post hoc test. (D) Scheme for exophilin-5 regulation of pathogenic Th2 cell functions. See text in Discussion.

kine production by enhancing pathway(s) downstream of IL-33R in addition to enhancing IL-33R expression itself (Figure 10).

Consistent with the phenotypes of exophilin-5 deficiency, Rab27a deficiency enhanced IL-5 production in the presence of IL-33 and decreased extracellular superoxide secretion by $\mathrm{CD}^{+} \mathrm{T}$ cells upon TCR stimulation. A genetic link between RAB27A SNPs and FeNo level, but not with asthma or atopy itself was reported in humans (15). Considering that exophilin-5 deficiency in pathogenic Th2 cells exacerbates allergic lung inflammation after presensitization, but not in the antigen sensitization phase, in mice, Rab27a dysfunctions in those cells may also aggravate eosinophilic lung inflammation (detectable as increased FeNO levels) by enhancing IL-5 and IL-13 production in antigen-sensitized individuals, but not by increasing the onset of asthma or atopy. In this context, SNPs in Rab27a may predict responsiveness in asthmatics to anti-IL-5 or anti-IL-13 neutralizing antibody. These possibilities merit further examination.

In summary, the present study reveals the unappreciated regulatory mechanism of allergic lung inflammation controlled by the Rab27a effector, exophilin- 5 . Our findings suggest that exophilin-5 regulates allergic lung inflammation by controlling NADPH oxidase

requires further characterization in a future study. These cells specifically coexpressed high levels of exophilin-5 and IL-33R. Furthermore, expression levels of exophilin-5-encoding EXPH5 and IL-33R-encoding IL1RL1 mRNAs were well correlated in human memory-type Th2-enriched $\mathrm{CD} 4{ }^{+} \mathrm{CCR} 4^{+} \mathrm{T}$ cells, suggesting a strong functional connection between these 2 proteins. Consistently, exophilin-5 deficiency significantly increased IL-5 and IL-13 production in response to IL-33 and TCR stimulation, with upregulation of IL-33R in pathogenic Th2 cells. Considering that the NADPH oxidase inhibitor DPI suppressed IL- 5 and IL-13 production more robustly than IL-33R expression in pathogenic Th2 cells, exophilin-5 deficiency possibly enhances IL-33-dependent cyto- localization and, thereby, IL-33-induced IL-5 and IL-13 production, in pathogenic Th2 cells. These findings may contribute to the development of new therapeutic strategies in the treatment of asthma, such as by targeting Rab27-related molecules.

\section{Methods}

Additional details can be found in the supplemental methods.

Generation of exophilin-5-deficient mice. C57BL/6N mice were purchased from CLEA Japan. The Exph5-KO mice (accession no. CDB0982K: http://www2.clst.riken.jp/arg/mutant\%20mice\%20list. $\mathrm{htm}$ ) were generated as described elsewhere (49). To construct a targeting vector, genomic fragments of the exophilin-5 locus were obtained 
from a BAC clone (BACPAC Resources). Exon6 of the Exph5 gene was disrupted by insertion of a loxP-flanked cassette of the neomycin resistance gene under the control of the phosphoglycerate kinase (PGK) promoter (Figure 1A). Targeted TT2 (derived from F1 of C57BL/6 and CBA) embryonic stem cell clones (50) were microinjected into 8-cellstage ICR embryos, and were then transferred into pseudopregnant ICR females. The resulting chimeras were bred with C57BL/6 mice, and heterozygous offspring were identified by Southern blotting and polymerase chain reaction (PCR). The primers used for PCR were Exo5/Fow1 (5'-TTGGGAGCCCCAGCTCAGCT-3') and Exo5/Rev1 (5'-CTGAGAAGTGGCGCCCCCTG-3') for the WT allele, and Neo/ Fow2 (5'-CATGCCCGACGGCGAGGATC-3) and Exo5/Rev1 for the targeted allele. Sizes of PCR products for WT and Exph5-KO mice are 268 bp and 809 bp, respectively. Mutant lines were backcrossed with C57BL/6N mice for 11 generations. Lack of exophilin-5 protein in Exph5-KO mice was confirmed by immunoblotting. For immunoblotting, isolated cerebellum from WT mice and Exph5-KO mice were lysed with buffer (20 mM HEPES pH 7.4, $150 \mathrm{mM} \mathrm{NaCl}$, 1\% Triton X-100, 0.2 $\mathrm{mM}$ EDTA, and $1 \mathrm{mM}$ dithiothreitol) containing protease and phosphatase inhibitors. The protein extracts $(50 \mu \mathrm{g})$ were loaded onto $5 \%$ polyacrylamide gels for electrophoresis. Rabbit anti-exophilin-5 antibody was raised against a GST-fused C-terminal region (aa 1401-1600) of exophilin-5 protein, as described previously (51). The sera were passed through a column containing GST protein, followed by one containing the GST-fused corresponding region of exophilin-5 protein. The affinity-purified antibodies were then eluted and concentrated.

Animals. Il33-KO mice (accession no. CDB0631K) were generated and backcrossed with C57BL/6N, as reported previously (52). Exph5 and Il33 DKO mice were obtained by crossing Exph5-KO mice with Il33-KO mice. The Rab27a-mutated ashen mice (C3H/He background) and control $\mathrm{C} 3 \mathrm{H} / \mathrm{He}$ mice were provided by N.A. Jenkins (National Cancer Institute, Frederick, Maryland, USA). Male mice were phenotypically characterized in the present study unless otherwise indicated. Mice had ad libitum access to water and standard laboratory chow (CE-2; CLEA Japan) in an air-conditioned room with 12-hour light/dark cycles. For BM transplantation, recipient C57BL/6N mice, between the ages of 8 and 10 weeks, were irradiated twice with an individual dose of 5.4 Gy with a 3-hour interval, and subsequently received an injection of $2 \times 10^{6} \mathrm{BM}$ cells from the tail vein.

OVA-induced asthma protocol. An OVA-induced mouse model of asthma was developed as described previously $(53,54)$, with slight modifications. In brief, mice at the ages of 8 to 12 weeks or 6 to 10 weeks after the BM transfer were sensitized with $20 \mu \mathrm{g}$ of OVA (MilliporeSigma) mixed with $2 \mathrm{mg}$ of alum (Thermo Fisher Scientific) intraperitoneally on days 0 and 10 . In some experiments, on day 17, spleen cells, splenic T cells, or splenic DCs were harvested as described below, and then analyzed further. Otherwise, mice were challenged with $3 \%(\mathrm{w} / \mathrm{v})$ OVA aerosol in physiologic saline delivered by nebulizer for 10 minutes every day on days 18 to 20. This well-established protocol is known to result in eosinophilic inflammation and induction of Th2 cytokines in BALF. Control mice received the saline aerosol delivered by a nebulizer for 10 minutes on days 18 to 20 . Twelve hours (for measurement of cytokines in BALF and lungs) or 24 hours (for the others) after the final challenge, BALF, thoracic lymph nodes, and lung tissues were obtained for the further analysis.

Intratracheal injections of Alternaria extract. Mice were anesthetized with intraperitoneal injection of ketamine and xylazine, and trachea of each mice were exposed. Next, $50 \mu \mathrm{L}$ of PBS or Alternaria extract $(10 \mu \mathrm{g}$ in $50 \mu \mathrm{L}$ PBS, ITEA Inc.) was injected into the mouse lung through the trachea using a $26-\mathrm{G}$ needle and $1-\mathrm{mL}$ syringe. Thirty minutes later, BALF and lung samples were harvested.

House dust mite-induced asthma protocol. Mice received intranasal administrations of $20 \mu \mathrm{L}$ of house dust mite extract $(1 \mathrm{mg} / \mathrm{mL})$ derived from Dermatophagoides farinae (Greer Laboratories) or $20 \mu \mathrm{L}$ of PBS alone under isoflurane general anesthesia every 2 to 3 days (3 times/ week). Twenty-four hours after the seventh intranasal administration, BALF samples were harvested.

$B A L F$ analyses. BALF analyses were performed as described previously $(53,54)$. In brief, the lungs were lavaged 4 times with PBS (0.5 $\mathrm{mL}$ each) for cell analysis. The cell suspension was centrifuged 500 $g$ for 5 minutes at $4^{\circ} \mathrm{C}$, cells were resuspended in $1 \mathrm{~mL}$ of physiological saline with 1\% BSA (Wako), and the total cell number was counted with a hemocytometer. Cytospin samples were prepared by centrifuging the suspensions at $500 \mathrm{rpm}$ for 3 minutes. Based on the findings obtained with Diff-Quik staining (Kokusai-Shiyaku), cell differentials were counted with at least 300 leukocytes in each sample. For measurement of cytokines, lungs were repeatedly lavaged 2 times with 0.4 $\mathrm{mL}$ of PBS, and after centrifugation of BALF, supernatants were collected and subjected to measurement of cytokine concentrations by ELISA. LDH activity in BALF was determined by a Cytotoxicity Detection Kit (Roche).

ELISA. Concentrations of mouse IL-4, IL-5, IFN- $\gamma$ (BD Biosciences), IL-13, IL-33, TSLP, soluble ST2 (eBioscience), IL-1 $\alpha$ (BioLegend), and IL-25 (R\&D Systems) were measured using the ELISA kits, following the manufacturers' protocols.

Cytokine concentration in the lung homogenates. The left lungs were homogenized in $1.0 \mathrm{~mL}$ PBS containing 0.5\% Triton X-100 and complete protease inhibitor mixture (Roche). The lung homogenates were cleared of debris and cells by centrifugation at 10,000 $\mathrm{g}$ for $10 \mathrm{~min}-$ utes. Cytokine concentrations in the lung homogenate were measured by an ELISA, and then normalized to the total protein concentrations in the homogenates.

Histopathological examination. For detection of mucus production in the lung epithelium, formalin-fixed, paraffin-embedded right lung sections cut at a thickness of $2 \mu \mathrm{m}$ were stained with periodic acidSchiff (PAS). Images were acquired on a BX51 Olympus microscope using DP2-BSW software.

OVA-specific spleen cell and lymph node cell responses. Spleens and thoracic lymph node cells were collected, transferred into Petri dishes, and repeatedly injected at different sites with a $26-\mathrm{G}$ needle with $1 \mathrm{mg} /$ $\mathrm{mL}$ collagenase I (Invitrogen) in RPMI media until $2 \mathrm{~mL}$ was injected (53-55). Tissues were then incubated at $37^{\circ} \mathrm{C}$ for 12 minutes and subsequently minced with the back of the syringe. Single-cell suspensions were prepared by passing through a $40-\mu \mathrm{m}$ cell strainer (BD Biosciences). Erythrolysis was performed with erythrocyte lysing buffer consisting of $155 \mathrm{mM} \mathrm{NH}_{4} \mathrm{Cl}, 5.7 \mathrm{mM} \mathrm{K}_{2} \mathrm{HPO}_{4}$, and $0.1 \mathrm{mM}$ EDTA at room temperature for 1 minute, and stopped by addition of RPMI medium. Splenocytes or lymph node cells $\left(6 \times 10^{5}\right.$ cells/well, total $150 \mu \mathrm{L} /$ well $)$ were suspended in complete RPMI medium (RPMI1640 containing $10 \%$ FBS and penicillin/streptomycin), and cultured in a flat-bottom 96-well plate in the presence and absence of OVA $(1 \mathrm{mg} / \mathrm{mL}$, unless otherwise indicated) at $37^{\circ} \mathrm{C}$ for the indicated number of days.

Measurement of airway hyperresponsiveness. At 24 hours after the final OVA aerosol challenge, airway hyperresponsiveness to metha- 
choline (MilliporeSigma) was measured as described elsewhere (56). Briefly, mice were deeply anesthetized with ketamine (Daiichi-Sankyo) and xylazine (Nihon-zenyaku), and then were tracheostomized and connected to plethysmograph chambers with a ventilator (Elan Series Mouse RC Site; Buxco Electronics). After 1 minute of nebulization with PBS or methacholine $(0.78-3.125 \mathrm{mg} / \mathrm{mL})$, lung resistance $\left(\mathrm{R}_{\mathrm{L}}\right)$ was continuously monitored for 3 minutes and the average of $\mathrm{R}_{\mathrm{L}}$ in each period was calculated using BioSystem XA software (Buxco Electronics). $\mathrm{R}_{\mathrm{L}}$ values were normalized to that obtained after PBS nebulization in each mouse.

Flow cytometry. For flow cytometric staining, cells were resuspended in PBS containing $2 \mathrm{mM}$ EDTA and 2\% FBS. Fc receptormediated nonspecific antibody binding was blocked by the addition of excessive amounts of anti-mouse CD16/CD32 monoclonal antibody (2.4G2). Staining was performed at $4^{\circ} \mathrm{C}$ in the dark for 20 minutes. The fluorochrome-conjugated monoclonal antibodies listed in Supplemental Table 1 were used at appropriate dilutions for staining of cell surface molecules. Some cells were stained as negative controls with fluorochrome-matched isotype-control antibodies. For detection of IL-33R, after confirmation that antibodies sourced from 2 different companies provided similar results, we mainly used PE-conjugated anti-mouse IL-33R monoclonal antibody purchased from eBioscience. After excluding dead cells by staining with 7-aminoactinomycin D (7-AAD) (for nonfixed cells, BD Biosciences) or Fixable Viability Dye eFluor 450 (for intracellular staining, eBioscience), live cells were subjected to characterization of cell populations or to sorting of specific cell populations using a FACSVerse or FACSAria II flow cytometer (BD Biosciences). For detection of intracellular proteins, cell surface molecules and dead cells were first stained. Next, cells were fixed and permeabilized, and intracellular proteins were stained with antibodies using the Intracellular Fixation \& Permeabilization Buffer Set (eBioscience), following the manufacturer's instructions.

Isolation of 4 different populations in splenic $C D 4^{+} C D 44^{h i}$ memory-type Th cells. For sorting of 4 different subpopulations in splenic $\mathrm{CD} 4{ }^{+} \mathrm{CD} 44^{\text {hi }}$ memory-type Th cells, $\mathrm{CD} 4^{+}$Th cells were first enriched using microbead-conjugated anti-CD4 (IMag-mouse CD4, BD Biosciences, catalog 551539), and were stained with fluorochrome-conjugated monoclonal antibodies as follows: $\mathrm{PE}-\mathrm{Cy} 7-$ conjugated anti-mouse $\mathrm{CD} 3 \varepsilon$, FITC-conjugated anti-mouse CD4, APC-Cy7-conjugated antimouse CD44, APC-conjugated anti-mouse CXCR3, and PE-conjugated anti-mouse CD62L. Then, 4 populations were sorted based on levels of cell surface CD62L and CXCR3, as previously reported by others (24). For functional analysis, considering that the purities of $\mathrm{CD}^{+}$and $\mathrm{CD}^{+} \mathrm{CD}^{+}$cells sorted using microbead-conjugated anti-CD4 were as high as approximately $99 \%$ and approximately $95 \%$, respectively, and to avoid unnecessary activation and interference of subsequent TCR-mediated activation of T cells by fluorochrome-conjugated antiCD3 $\varepsilon$ antibody, we sorted 4 fractions from magnetically sorted CD $4^{+}$ T cells without staining either CD3 or CD4.

Isolation of other immune cells. For isolation of CD11 $\mathrm{c}^{+}$splenic DCs, after blocking with anti-CD16/CD32 monoclonal antibody, splenocytes were incubated with magnetic bead-conjugated anti-mouse CD11c antibodies (Miltenyi Biotec, catalog 130-052-001) followed by magnetic separation using an LS column according to the manufacturer's instructions. The purity of CD11 $\mathrm{c}^{+}$cells in live cells, confirmed by flow cytometry, was approximately $85 \%$. For isolation of BMderived basophils and mast cells, BM cells obtained from WT mice were cultured in complete RPMI media containing recombinant mouse IL-3 (PeproTech) at $2 \mathrm{ng} / \mathrm{mL}$ for 7 days. On days 3 and 6, media were completely refreshed with IL-3-containing media. On day 7, basophils and mast cells were sorted as FceRIa $\alpha^{+} \mathrm{CD} 49 \mathrm{~b}^{+} \mathrm{CD} 117^{-}$cells and $\mathrm{FcERI} \alpha^{+} \mathrm{CD} 49 \mathrm{~b}^{-} \mathrm{CD} 117^{+}$cells, respectively. BM-derived macrophages were harvested as previously described (57). CD11b $\mathrm{b}^{+}$macrophages were enriched from cells obtained from WT mice by peritoneal lavage using magnetic bead-conjugated anti-mouse CD11b antibodies (Miltenyi Biotec, catalog 130-049-601). Eosinophils were isolated as described previously (58), with slight modification. One hundred micrograms of the plasmid pCAGGS-IL-5 in lactated Ringer's solution $(0.1 \mathrm{~mL} / \mathrm{g}$ body weight) was injected into the tail veins of mice. Two weeks after the injections, siglec- $\mathrm{F}^{+}$splenocytes were sorted as eosinophils. For isolation of neutrophils, mice received intraperitoneal injections of $500 \mu \mathrm{L}$ of saline containing $50 \mathrm{ng}$ of lipopolysaccharide. Six hours after the injections, peritoneal cells were harvested and neutrophil-enriched fractions with purity of neutrophils at or above $70 \%$ (determined by cytospin) were used for analysis.

Isolation of epidermal cells, alveolar epithelial cells, and lung $\mathrm{CD}^{+} \mathrm{Th}$ cells. Epidermal cells were isolated using an epidermal isolation kit and gentleMACS Dissociator (Miltenyi Biotec), following the manufacturer's instructions. Lung epithelial cells were isolated as previously reported (59) with slight modifications. In brief, lungs were expanded with intratracheal injection of $2.5 \mathrm{~mL}$ of physiologic saline containing Dispase (Invitrogen) at $28 \mathrm{mg} / \mathrm{mL}$, and were left at room temperature for 30 minutes. Next, lung cells were gently minced with forceps, and were rocked in $10 \mathrm{~mL}$ of PBS containing 0.01\% DNase I (Roche) and $0.5 \% \mathrm{BSA}$ at $200 \mathrm{rpm}$ for 10 minutes at room temperature. Then, after serially filtering through $150-\mu \mathrm{m}$ and $40-\mu \mathrm{m}$ mesh, the cell suspensions were subjected to red blood cell lysis, and were then stained with anti-CD45-FITC and anti-CD326-PE or -APC for sorting of $\mathrm{CD}^{-} 5^{-} \mathrm{CD} 326^{+}$lung epithelial cells and $\mathrm{CD} 45^{+} \mathrm{CD} 326^{-}$lung leukocytes using a FACSAria II. Lung $\mathrm{CD}^{+}{ }^{+} \mathrm{Th}$ cells were isolated using microbead-conjugated anti-CD4 as described above.

Ex vivo activation of $C D 4^{+} T$ cells. Ninety-six-well plates were coated with $1 \mu \mathrm{g} / \mathrm{mL}$ anti-CD3ع (BioLegend) antibodies overnight at $4^{\circ} \mathrm{C}$ and washed with complete media to remove unbound soluble antibodies. Splenic $\mathrm{CD}^{+} \mathrm{T}$ cells or sorted subpopulations were plated at $1.5 \times$ $10^{5}$ cells (unless otherwise indicated) in $150 \mu \mathrm{L}$ per well in the presence or absence of $2 \mathrm{ng} / \mathrm{mL} \mathrm{IL-33}$, and were then cultured for 2 days. In some experiments, cells were plated after 10 minutes of preincubation with the indicated kinase inhibitors. The optimal concentration of each inhibitor was determined based on our preliminary experiments. Then, after centrifugation of culture plates at $500 \mathrm{~g}$ for 5 minutes at $4^{\circ} \mathrm{C}$, culture supernatants were harvested. In some experiments, after removal of supernatants and 2 washes with PBS, cell lysates were collected in $150 \mu \mathrm{L}$ of lysis buffer used for lung homogenates. Supernatants and cell lysates were subsequently analyzed by ELISA.

Splenic $\mathrm{CD}^{+} \mathrm{T}$ cell transfer. $\mathrm{CD} 4^{+} \mathrm{T}$ cells were isolated from spleens of OVA-sensitized mice using magnetic beads for $\mathrm{CD} 4^{+}$selection as described above. Based on our preliminary experiments, to obtain mild allergic airway inflammation, we used female recipient mice that received 1 OVA/alum intraperitoneal injection 7 days before $\mathrm{CD}^{+} \mathrm{T}$ cell transfer. Five million $\mathrm{CD} 4^{+} \mathrm{T}$ cells were injected intravenously into the recipient mice, and 24 hours later the mice were challenged with $3 \%$ OVA for 3 consecutive days; 24 hours after the last airway challenge the mice were sacrificed and samples were collected. 
$R N A$ preparation and gene expression analyses for murine samples. RNA was extracted using Sepasol-RNA I Super (Nacalai Tesque). Total RNA $(1 \mu \mathrm{g})$ was reverse transcribed using oligo-(dT)12-18 primer and Superscript III (Invitrogen). Quantitative PCR was performed with SYBR premix Ex Taq (Takara Bio) using a C1000 Thermal Cycler (Bio-Rad). The results were normalized against $18 \mathrm{~S}$ rRNA or RplpO/36B4 mRNA expression. The primer sequences used are listed in Supplemental Table 2.

Isolation of human memory-type Th2 cell-enriched fraction. For Th2 cell isolation, cells from human peripheral blood were drawn from 12 voluntary subjects ( 3 females and 9 males, ages 26-65), and lymphocytes were separated by density gradient centrifugation using FicollPaque PLUS (GE Healthcare). Then, using a CD4 ${ }^{+} \mathrm{T}$ cell Isolation kit (Miltenyi Biotec, catalog 130-096-533), CD4 ${ }^{+}$cells were collected by negative selection according to the manufacturer's instructions. Next, the cells were labeled with PE-conjugated anti-CCR4 antibody (mouse IgG1k, clone L291H4, BioLegend, catalog 359411) followed by incubation with anti-PE MicroBeads (Miltenyi Biotec, catalog 130-048-801). Cells were finally positively selected and the purities of the cell population were assessed by BD FACSVerse. The purities of $\mathrm{CD} 4^{+} \mathrm{CCR} 4^{+}$ cells in sorted cells in 12 individuals were $82.2 \% \pm 1.8 \%$ (mean \pm SEM).

Real-time quantitative PCR for human $\mathrm{CD} 4^{+} \mathrm{CCR} 4^{+}$cells. Total RNA was extracted from isolated cells with an RNeasy Mini Kit (Qiagen) according to the manufacturer's instructions. The extracted mRNA was reverse transcribed to cDNA using an iScript cDNA Synthesis Kit (Bio-Rad). Real-time quantitative PCR analysis was performed using a Probe qPCR Mix (Takara Bio) and StepOnePlus Real-time PCR System (Applied Biosystems). The primers and probes for human $\beta$-actin-encoding ACTB, EXPH5, and IL1RL1 were designed by Applied Biosystems (assay IDs for the indicated genes are Hs01060665_g1, Hs00323579_m1, and Hs00249384_m1, respectively). Values of target mRNA levels relative to those of $A C T B$ were calculated by the $\Delta \mathrm{Ct}$ method using the following equation: $R Q=2^{-\Delta C t}$.

Plasmid construction. Full-length cDNAs encoding mouse exophilin-5, Rab27a, and Nox2 were amplified from mouse cDNAs by PCR using the following pairs of oligonucleotides with a BamHI linker, an EcoRI linker, or an XhoI linker: 5'-GGGGATCCATGACGAAAGTTCCTCAGGG-3' and 5'-GGCTCGAGTCATAGTTCTGACTCTTTATCC-3' for exophilin-5; 5'-GGGAATTCATGTCGGATGGAGATTAC-3' and 5'-GGCTCGAGTCAACAGCCACACAACCC-3' for Rab27a; and 5'- GGGAATTCATGGGGAACTGGGCTGTG-3' and 5'-GGCTCGAgtTAgAagTTTTCCTTGTT-3' for Nox2. Purified PCR products were subcloned into the BamHI, EcoRI, and XhoI sites of pcDNA3-FLAG tag, pcDNA3-HA tag, and pEGFP-C1 vectors. Plasmid DNA for transfection into mammalian cell cultures was prepared using a Promega miniprep kit following the manufacturer's instructions.

Plasmid transfection. HEK293A cells were cultured in DMEM supplemented with $10 \%$ FBS, 100 units/mL penicillin G, and 100 $\mu \mathrm{g} / \mathrm{mL}$ streptomycin at $37^{\circ} \mathrm{C}$ in $5 \% \mathrm{CO}_{2}$. FLAG-Nox2 and HA-Rab27a (a total of $4 \mu \mathrm{g}$ plasmid), or FLAG-exophilin-5, GFP-Nox2, and HARab27a (a total of $8 \mu \mathrm{g}$ plasmid) were transfected into HEK293A cells using Lipofectamine 2000 reagent (Thermo Fisher Scientific). Two days after transfection, cells were harvested and homogenized. For plasmid transfection into $\mathrm{CD} 4^{+} \mathrm{Th}$ cells, untouched $\mathrm{CD} 4^{+} \mathrm{T}$ cells were enriched using an IMag Mouse CD4 T Lymphocyte Enrichment Set (BD Biosciences, catalog 558131), and then GFP-Nox2 and HARab27a ( $2 \mu$ g plasmid each) were transfected into those cells by elec- troporation using an Amaxa Mouse T cell Nucleofector Kit (Lonza), following the manufacturer's instruction.

Immunoprecipitation and immunostaining. For immunoprecipitation, HEK293A cell lysates were incubated with anti-FLAG affinity gel (30 $\mu \mathrm{L}$; MilliporeSigma) with gentle agitation at $4^{\circ} \mathrm{C}$ for 1 hour. After the beads were washed 5 times with lysis buffer, the precipitated proteins were separated by SDS-PAGE. For immunostaining of pathogenic Th2 cells, cells attached on slide glasses (Thermo Fisher Scientific) by cytospin at $800 \mathrm{rpm}$ for 2 minutes at room temperature were fixed with $3 \%$ paraformaldehyde for 30 minutes at room temperature and quenched for 5 minutes with $50 \mathrm{mM} \mathrm{NH}_{4} \mathrm{Cl}$. Fixed cells were washed with PBS and permeabilized with $0.1 \%$ Triton X-100 for 20 minutes, blocked with 1\% BSA in PBS for 30 minutes, incubated with anti-mouse Nox2 antibody derived from rabbit (Abcam) overnight, washed, and stained for 60 minutes with Alexa Fluor 488-conjugated secondary antibody. For detection of HA-Rab27a in CD4 ${ }^{+} \mathrm{T}$ cells transfected with GFPNOX and HA-Rab27a, cells were incubated first with rat-derived primary antibody against HA (clone 3F10, Roche), followed by anti-ratAlexa Fluor 568 secondary antibody. After a 15-minute staining with DAPI and 5 washes with PBS, coverslips were mounted in SlowFade Gold antifade reagent (Invitrogen). Stained cells were analyzed using an A1 confocal microscope (Nikon) equipped with a 100× oil immersion objective lens (1.49 numerical aperture) and NIS-Elements software. Detailed information regarding antibodies used in this paragraph are listed in Supplemental Table 3.

Detection of extracellular superoxide secretion. Pathogenic Th2 cells were sorted as described above. Next, $2 \times 10^{5}$ cells were resuspended in $20 \mu \mathrm{L}$ of complete RPMI media. Extracellular superoxide secretion after PMA and ionomycin stimulation (at $0.24 \mu \mathrm{g} / \mathrm{mL}$ each) was detected using the Diogenes Cellular Luminescence Enhancement System (National Diagnostics). The light units were normalized to the average values in WT cells at 2 minutes after PMA and ionomycin stimulation.

Detection of intracellular ROS. Sorted pathogenic Th2 cells were cultured at $1.5 \times 10^{5}$ cells in $75 \mu \mathrm{L}$ of complete RPMI media per well in a 96-well plate in the presence and absence of precoated anti-CD3 $\varepsilon$ antibody for 1 hour. Next, $75 \mu \mathrm{L}$ of complete RPMI media containing the ROS sensor CellROX Green Reagent (Invitrogen) was added into each well, and cells were incubated for a further 30 minutes. Then, cells were collected, stained with 7-AAD after 2 washes with FACS staining buffer, and analyzed by FACS for detection of green fluorescence-positive (intracellular ROS-positive) cells.

Statistics. All data are displayed as mean values \pm SEM (for $n=3$ or more) unless otherwise indicated. Figures were produced and statistics analyzed using Prism version 8 (GraphPad Software) or Microsoft Excel. Statistical differences among treatment groups were estimated by ANOVA or by repeated-measures ANOVA with Tukey's post hoc test for multiple comparisons. A 2-tailed $t$ test was used for comparison of 2 groups. In all instances, statistical significance was inferred from a $P$ value of less than 0.05 .

Study approval. The protocols for mouse experiments were approved by the Institutional Animal Care and Use Committee of RIKEN Kobe Branch, and by the Animal Care and Experimentation Committee, Gunma University. The protocol for isolation of Th2 cells from human peripheral blood was approved by the IRB of National Hospital Organization Tokyo National Hospital (approval number 180059), and all participants provided written informed consent before their participation in this study. 


\section{Author contributions}

KO, HW, MS, and EK performed experiments and analyzed data. RI initially characterized exophilin-5 KO mice. MK and TA generated Exph5-KO mice. MH and AS analyzed data. HS and SN generated Il33-KO mice and analyzed data. JM constructed the plasmid pCAGGS-IL-5. KO and TI designed experiments, wrote the manuscript, and supervised the research. KO and HW equally contributed to the data production and thus are credited as co-first authors. However, KO was chosen as the first name, because he played much more fundamental roles in designation of the experiments, supervision of the project, and writing the manuscript.

\section{Acknowledgments}

The authors thank the members of the Laboratory of Molecular Endocrinology and Metabolism, Gunma University, particularly T. Nara for her technical assistance and for colony maintenance of mice, T. Ushigome for colony maintenance of mice, and S. Shigoka for general management. The authors also thank Y. Yoshida at Heavy Ion Medical Center, Gunma University, for technical assistance in establishment of bone marrow-chimeric mice, the staff at Bioresource Center, Gunma University, for their help in breeding of mice, K. Moro and Y. Motomura at Osaka University for their suggestions in establishment of mouse lung ILC2 isolation, and S. Igarashi at National Hospital Organization Tokyo National Hospital for her technical assistance in isolation and real-time RT-PCR analysis of human $\mathrm{CD}^{+}{ }^{+} \mathrm{CCR} 4{ }^{+}$cells. This work was supported by JSPS KAKENHI grant numbers 26461484 and 17K09994 (to KO), as well as 20113005, 14F04104, and 19H03449 (to TI). It was also supported by grants from Takeda Science Foundation (to KO) and Novartis Pharma (to $\mathrm{KO}$ and $\mathrm{TI}$ ), and by the joint research program of the Institute for Molecular and Cellular Regulation, Gunma University (grants 14018 and 17003 to MS, 15014 and 18017 to AS, and 16002 to SN).

Address correspondence to: Katsuhide Okunishi, 3-39-15 Showamachi, Maebashi, Gunma 371-8512, Japan. Phone: 81.27.220.8877; Email: okunishik@gunma-u.ac.jp. Or to: Tetsuro Izumi, 3-3915 Showa-machi, Maebashi, Gunma 371-8512, Japan. Phone: 81.27.220.8856; Email: tizumi@gunma-u.ac.jp.
1. Holt PG, Macaubas C, Stumbles PA, Sly PD. The role of allergy in the development of asthma. Nature. 1999;402(6760 suppl):B12-B17.

2. Global Asthma Network. The Global Asthma Report 2018. Auckland, New Zealand: Global Asthma Network; 2018.

3. Izumi T. Physiological roles of Rab27 effectors in regulated exocytosis. Endocr J. 2007;54(5):649-657.

4. Yi Z, et al. The Rab27a/granuphilin complex regulates the exocytosis of insulin-containing dense-core granules. Mol Cell Biol. 2002;22(6):1858-1867.

5 . Hume AN, et al. The leaden gene product is required with Rab27a to recruit myosin Va to melanosomes in melanocytes. Traffic. 2002;3(3):193-202.

6. Stinchcombe JC, et al. Rab27a is required for regulated secretion in cytotoxic T lymphocytes. J Cell Biol. 2001;152(4):825-834.

7. Haddad EK, Wu X, Hammer JA, Henkart PA. Defective granule exocytosis in Rab27adeficient lymphocytes from Ashen mice. J Cell Biol. 2001;152(4):835-842.

8. Menasche G, et al. Mutations in RAB27A cause Griscelli syndrome associated with haemophagocytic syndrome. Nat Genet. 2000;25(2):173-176.

9. Johnson JL, et al. Rab27a and Rab27b regulate neutrophil azurophilic granule exocytosis and NADPH oxidase activity by independent mechanisms. Traffic. 2010;11(4):533-547.

10. Ejlerskov P, et al. NADPH oxidase is internalized by clathrin-coated pits and localizes to a Rab27A/B GTPase-regulated secretory compartment in activated macrophages. J Biol Chem. 2012;287(7):4835-4852.

11. Elstak ED, et al. The munc13-4-rab27 complex is specifically required for tethering secretory lysosomes at the plasma membrane. Blood. 2011;118(6):1570-1578.

12. Singh RK, et al. Distinct and opposing roles for Rab27a/Mlph/MyoVa and Rab27b/Munc13-4 in mast cell secretion. FEBS J. 2013;280(3):892-903.
13. Jancic C, et al. Rab27a regulates phagosomal $\mathrm{pH}$ and NADPH oxidase recruitment to dendritic cell phagosomes. Nat Cell Biol. 2007;9(4):367-378.

14. Bolasco G, et al. Loss of Rab27 function results in abnormal lung epithelium structure in mice. $A m J$ Physiol Cell Physiol. 2011;300(3): C466-C476.

15. Bouzigon E, et al. A common variant in RAB27A gene is associated with fractional exhaled nitric oxide levels in adults. Clin Exp Allergy. 2015;45(4):797-806.

16. Dweik RA, et al. An official ATS clinical practice guideline: interpretation of exhaled nitric oxide levels (FENO) for clinical applications. Am J Respir Crit Care Med. 2011;184(5):602-615.

17. McGrath JA, et al. Germline mutation in EXPH5 implicates the Rab27B effector protein Slac2-b in inherited skin fragility. Am J Hum Genet. 2012;91(6):1115-1121.

18. Ostrowski M, et al. Rab27a and Rab27b control different steps of the exosome secretion pathway. Nat Cell Biol. 2010;12(1):19-30.

19. Gerber PP, et al. Rab27a controls HIV-1 assembly by regulating plasma membrane levels of phosphatidylinositol 4,5-bisphosphate. JCell Biol. 2015;209(3):435-452.

20. Seumois G, et al. Transcriptional profiling of Th2 cells identifies pathogenic features associated with asthma. J Immunol. 2016;197(2):655-664.

21. Lefrançais E, Cayrol C. Mechanisms of IL-33 processing and secretion: differences and similarities between IL-1 family members. Eur Cytokine Netw. 2012;23(4):120-127.

22. Zhou X, Wei T, Cox CW, Jiang Y, Roche WR, Walls AF. Mast cell chymase impairs bronchial epithelium integrity by degrading cell junction molecules of epithelial cells. Allergy. 2019;74(7):1266-1276.

23. Galli SJ, Tsai M. IgE and mast cells in allergic disease. Nat Med. 2012;18(5):693-704.

24. Endo Y, et al. Eomesodermin controls interleukin- 5 production in memory $\mathrm{T}$ helper 2 cells through inhibition of activity of the transcription factor GATA3. Immunity. 2011;35(5):733-745.
25. Endo Y, Hirahara K, Yagi R, Tumes DJ, Nakayama T. Pathogenic memory type Th2 cells in allergic inflammation. Trends Immunol. 2014;35(2):69-78.

26. Onodera A, Kokubo K, Nakayama T. Epigenetic and transcriptional regulation in the induction, maintenance, heterogeneity, and recall-response of effector and memory Th2 cells. Front Immunol. 2018;9:2929.

27. Endo Y, et al. The interleukin-33-p38 kinase axis confers memory $\mathrm{T}$ helper 2 cell pathogenicity in the airway. Immunity. 2015;42(2):294-308.

28. Guo L, et al. IL-1 family members and STAT activators induce cytokine production by Th2, Th17, and Th1 cells. Proc Natl Acad Sci US A. 2009;106(32):13463-13468.

29. Kurowska-Stolarska M, et al. IL-33 induces antigen-specific IL- $5^{+} \mathrm{T}$ cells and promotes allergic-induced airway inflammation independent of IL-4. J Immunol. 2008;181(7):4780-4790.

30. Park CO, Kupper TS. The emerging role of resident memory $\mathrm{T}$ cells in protective immunity and inflammatory disease. Nat Med. 2015;21(7):688-697.

31. Griesenauer B, Paczesny S. The ST2/IL-33 axis in immune cells during inflammatory diseases. Front Immunol. 2017;8:475.

32. Nakatani T, et al. CCR4 memory CD4 ${ }^{+}$T lymphocytes are increased in peripheral blood and lesional skin from patients with atopic dermatitis. J Allergy Clin Immunol. 2001;107(2):353-358.

33. Yoshida C, et al. Bcr-Abl signaling through the PI-3/S6 kinase pathway inhibits nuclear translocation of the transcription factor Bach2, which represses the antiapoptotic factor heme oxygenase-1. Blood. 2007;109(3):1211-1219.

34. Yamashita M, Kuwahara M. The critical role of Bach2 in regulating type 2 chronic airway inflammation. Int Immunol. 2018;30(9):397-402.

35. Zoncu R, Efeyan A, Sabatini DM. mTOR: from growth signal integration to cancer, diabetes and ageing. Nat Rev Mol Cell Biol. 2011;12(1):21-35.

36. Chen $\mathrm{L}$, et al. Cadmium induction of reactive oxygen species activates the mTOR pathway, leading to neuronal cell death. Free Radic Biol 
Med. 2011;50(5):624-632.

37. Silva A, Gírio A, Cebola I, Santos CI, Antunes F, Barata JT. Intracellular reactive oxygen species are essential for PI3K/Akt/mTORdependent IL-7-mediated viability of T-cell acute lymphoblastic leukemia cells. Leukemia. 2011;25(6):960-967.

38. Jackson SH, Devadas S, Kwon J, Pinto LA, Williams MS. T cells express a phagocyte-type NADPH oxidase that is activated after T cell receptor stimulation. Nat Immunol. 2004;5(8):818-827.

39. Belikov AV, Schraven B, Simeoni L. TCRtriggered extracellular superoxide production is not required for T-cell activation. Cell Commun Signal. 2014;12:50.

40. Gomi H, Mori K, Itohara S, Izumi T. Rab27b is expressed in a wide range of exocytic cells and involved in the delivery of secretory granules near the plasma membrane. Mol Biol Cell. 2007;18(11):4377-4386.

41. Bedard K, Krause KH. The NOX family of ROS-generating NADPH oxidases: physiology and pathophysiology. Physiol Rev. 2007;87(1):245-313.

42. Kouro T, Takatsu K. IL-5- and eosinophil-mediated inflammation: from discovery to therapy. Int Immunol. 2009;21(12):1303-1309.

43. Lambrecht BN, Hammad H, Fahy JV. The cytokines of asthma. Immunity. 2019;50(4):975-991.
44. Bel EH, et al. Oral glucocorticoid-sparing effect of mepolizumab in eosinophilic asthma. $N$ EnglJ Med. 2014;371(13):1189-1197.

45. Ortega HG, et al. Mepolizumab treatment in patients with severe eosinophilic asthma. $N$ EnglJ Med. 2014;371(13):1198-1207.

46. Castro M, et al. Dupilumab efficacy and safety in moderate-to-severe uncontrolled asthma. $N$ Engl JMed. 2018;378(26):2486-2496.

47. Rabe KF, et al. Efficacy and safety of dupilumab in glucocorticoid-dependent severe asthma. N EnglJMed. 2018;378(26):2475-2485.

48. O'Garra A. Cytokines induce the development of functionally heterogeneous $\mathrm{T}$ helper cell subsets. Immunity. 1998;8(3):275-283.

49. Laboratory for Animal Resources and Genetic Engineering RIKEN Center for Biosystems Research Web Site. Methods. http://www2.clst.riken.jp/arg/ methods.html. Accessed April 21, 2020.

50. Yagi T, et al. A novel ES cell line, TT2, with high germline-differentiating potency. Anal Biochem. 1993;214(1):70-76.

51. Wang H, et al. The Rab27a effector exophilin7 promotes fusion of secretory granules that have not been docked to the plasma membrane. $\mathrm{Mol}$ Biol Cell. 2013;24(3):319-330.

52. Oboki K, et al. IL-33 is a crucial amplifier of innate rather than acquired immunity. Proc Natl Acad Sci U S A. 2010;107(43):18581-18586.
53. Okunishi K, et al. A novel role of hepatocyte growth factor as an immune regulator through suppressing dendritic cell function. J Immunol. 2005;175(7):4745-4753.

54. Okunishi K, Dohi M, Nakagome K, Tanaka R, Yamamoto K. A novel role of cysteinyl leukotrienes to promote dendritic cell activation in the antigen-induced immune responses in the lung. JImmunol. 2004;173(10):6393-6402.

55. Zasłona Z, et al. Prostaglandin $\mathrm{E}_{2}$ suppresses allergic sensitization and lung inflammation by targeting the E prostanoid 2 receptor on T cells. JAllergy Clin Immunol. 2014;133(2):379-387.

56. Nakae S, et al. Mast cell-derived TNF contributes to airway hyperreactivity, inflammation, and TH2 cytokine production in an asthma model in mice. J Allergy Clin Immunol. 2007;120(1):48-55.

57. Zaslona Z, Serezani CH, Okunishi K, Aronoff DM, Peters-Golden M. Prostaglandin E2 restrains macrophage maturation via $\mathrm{E}$ prostanoid receptor 2/protein kinase A signaling. Blood. 2012;119(10):2358-2367.

58. Nakagome K, et al. IL-5-induced hypereosinophilia suppresses the antigen-induced immune response via a TGF-beta-dependent mechanism. JImmunol. 2007;179(1):284-294.

59. Demaio L, et al. Characterization of mouse alveolar epithelial cell monolayers. Am J Physiol Lung Cell Mol Physiol. 2009;296(6):L1051-L1058. 